\title{
Molecular docking of secondary metabolites from Indonesian marine and terrestrial organisms targeting SARS-CoV-2 ACE-2, Mpro, and PL ${ }^{\text {pro }}$ receptors
}

\author{
Gita Syahputra ${ }^{1}$, Nunik Gustini ${ }^{1}$, Bustanussalam Bustanussalam, Yatri Hapsari ${ }^{1}$, Martha Sari ${ }^{1}$, \\ Ardi Ardiansyah ${ }^{1}$, Asep Bayu ${ }^{1}$, Masteria Yunovilsa Putra ${ }^{1}$ \\ 1 Research Center for Biotechnology, Indonesian Institute of Sciences. Jl. Raya Jakarta-Bogor Km.46 Cibinong 16911, Indonesia \\ Corresponding authors: Gita Syahputra (gitasyahputra@gmail.com); Masteria Yunovilsa Putra (masteria.yunovilsa@gmail.com; mast001@lipi.go.id)
}

Received 9 May 2021 • Accepted 29 June 2021 • Published 23 July 2021

Citation: Syahputra G, Gustini N, Bustanussalam B, Hapsari Y, Sari M, Ardiansyah A, Bayu A, Putra MY (2021) Molecular docking of secondary metabolites from Indonesian marine and terrestrial organisms targeting SARS-CoV-2 ACE-2, $\mathrm{M}^{\text {pro }}$, and $\mathrm{PL}^{\text {pro }}$ receptors. Pharmacia 68(3): 533-560. https://doi.org/10.3897/pharmacia.68.e68432

\begin{abstract}
With the uncontrolled spread of severe acute respiratory syndrome coronavirus 2 (SARS-CoV-2), development and distribution of antiviral drugs and vaccines have gained tremendous importance. This study focused on two viral proteases namely main protease $\left(\mathrm{M}^{\mathrm{pro}}\right)$ and papain-like protease $\left(\mathrm{PL}^{\mathrm{pro}}\right)$ and human angiotensin-converting enzyme (ACE-2) to identify which of these are essential for viral replication. We screened 102 secondary metabolites against SARS-CoV-2 isolated from 36 terrestrial plants and 36 marine organisms from Indonesian biodiversity. These organisms are typically presumed to have antiviral effects, and some of them have been used as an immunomodulatory activity in traditional medicine. For the molecular docking procedure to obtain Gibbs free energy value $(\Delta \mathrm{G})$, toxicity, ADME and Lipinski, AutoDock Vina was used. In this study, five secondary metabolites, namely corilagin, dieckol, phlorofucofuroeckol $\mathrm{A}$, proanthocyanidins, and isovitexin, were found to inhibit $\mathrm{ACE}-2, \mathrm{M}^{\text {pro }}$, and $\mathrm{PL}^{\text {pro }}$ receptors in SARS-CoV-2, with a high affinity to the same sites of ptilidepsin, remdesivir, and chloroquine as the control molecules. This study was delimited to molecular docking without any validation by simulations concerned with molecular dynamics. The interactions with two viral proteases and human ACE-2 may play a key role in developing antiviral drugs for five active compounds. In future, we intend to investigate antiviral drugs and the mechanisms of action by in vitro study.
\end{abstract}

\section{Keywords}

Molecular docking, Secondary metabolites, Marine, Terrestrial, SARS CoV-2, Receptors

\section{Introduction}

The coronavirus disease (COVID-19), caused by severe acute respiratory syndrome coronavirus 2 (SARS-CoV-2) or the novel coronavirus, is a highly transmittable and pathogenic viral infection (Liu et al. 2020; Shereen et al. 2020) and the fifth pandemic after the 1918 flu pande- mic. The first cases of COVID-19 in humans caused by SARS-CoV-2 were detected in late December 2019 in Wuhan City, Hubei Province, China, and rapidly spread throughout the world in less than six months (Machhi et al. 2020; Tang et al. 2020). The most initial instances of COVID-19 in Indonesia were reported with two confirmed cases on March 2, 2020, and this number escalated 
to $1.52 \mathrm{M}$ by April 1, 2020 in all Indonesian provinces (Tosepu et al. 2020).

Numerous drugs and vaccines have been studied and developed further to fight SARS-CoV-2. Therefore, the secondary metabolites isolated from plants as the source of drug compounds have been examined and stated that they had potential antiviral activity to the group of coronavirus (Orhan and Deniz 2020). It is well-known that Indonesia has mega biodiversity and enormous biological resources in terms of flora, fauna, and macro- and microorganisms in the terrestrial region and ocean (Yap et al. 2019). The terrestrial and marine environments offer new abundant bioactive sources of molecules with unique structural features and various biological activities, such as antiviral, antimicrobial, antifungal, antiparasitic, antioxidant, antitubercular, anti-inflammatory, and antitumor (Terstappen and Reggiani 2001). The production of drug candidates from secondary metabolite sources to produce new bioactive compounds are often characterized by structural novelty, complexity, and diversity (Das et al. 2020; Lan et al. 2020).

It is known that SARS-CoV-2 mainly affects individuals with a weak immune system, and thus, enhancing immunity is one of the best ways to combat SARS-CoV-2. An integrated approach may not only enhance immunity, but also prevent any further complications (Dalan et al. 2020). Numerous researchers worldwide have expressed their interest in developing an effectual vaccine to combat COVID-19. Nigella sativa has been used for medicinal purposes for its intense immunoregulatory, anti-inflammatory, and antioxidant benefits in obstructive respiratory disorders. Research has found that Thymoquinone, Nigellidine, and $\alpha$-hederin from $N$. sativa can be potential influencers in reinforcing the immune response modulation at the molecular docking level (Hosseini et al. 2021). In a methodical research, docking was observed in gingerol, paradol, shogaol, and zingerone, and thus, they were deduced to be effective active compounds. Two compounds namely 6-gingerol and 10-gingerol formed covalent bonds with the enzyme, indicating that they are the most potent for drug development among the active components of ginger. The chemical structure of all the active components was drawn using an in silico prediction (Ibrahim et al. 2020). Exploring new medicines by investigating a large number of medicinal plants and identifying the active components in the treatment for SARS-CoV-2 can be accomplished through the interaction of compound drugs with the proteins associated with the diseases. An analysis with computer-aided drug design (bioinformatics) can provide meaningful and pertinent information. A study successfully demonstrated Indonesian medicinal plants' use to determine the potential candidate compounds effectual as supportive SARS-CoV-2 therapy. The researchers who conducted the study used molecular docking approach to analyze the interactions between the $3 \mathrm{CL}^{\text {pro }}$ protein (main protease) with 14 hit compounds from the chemical medicine database (PubChem (https://pubchem.ncbi.nlm.nih. gov/)). The same study affirmed six potential compounds as the main proteases of SARS-CoV-2 inhibitor. More spe- cifically, Hesperidin, Kaempferol-3,4'-di-O-methyl ether (Ermanin), Myricetin-3-glucoside, Peonidine 3-(4'-arabinosylglucoside), Quercetin 3-(2G-rhamnosylrutinoside), and Rhamnetin 3-mannosyl-(1-2)-alloside were demonstrated as potential candidates for antiviral for SARSCoV-2 (Naidoo et al. 2020).

An in silico approach can reduce the extensive exploration of numerous secondary metabolites to a fewer selected potential chemical compounds and is also efficacious as the initial step of drug discovery research (Terstappen and Reggiani 2001; Yap et al. 2019). Researchers worldwide are attempting to find adequate medication to combat the COVID-19 pandemic. Thus, investigating a potential compound for fighting SARS-CoV-2 by applying in silico antiviral peptides will assuredly be effectual to simplify rigorous experimentations (Fakih et al. 2020). The antiviral approaches consist of inhibiting the synthesis of an RNA virus and replicating the virus, thereby restricting the virus from binding to human cell receptors and obviating its self-forming process. Research had shown that when SARS-CoV-2 infected humans, the receptor-binding domain of the virus's spike protein was attached to angiotensin-converting enzyme (ACE-2) as the primary cell receptor (Das et al. 2020; Lan et al. 2020). ACE-2 is in charge of the renin-angiotensin system's major role to regulate blood pressure, fluid, and electrolyte balance. A previous study involving in vivo assay concluded that respiratory tract epithelia infected by SARS-CoV-2 were pertinent to the state of cell differentiation and ACE-2 expression. The infection is more likely to develop during sufficiently differentiated ciliated cells of increasing ACE-2 expression. The same research further showed that the expression of ACE-2 membrane and plasma level was decreased and the amplification of pulmonary wound after being infected by SARS-CoV-2 (Dalan et al. 2020). SARS-CoV-2 genomes encode 16 non-structural proteins. Two of them are the main protease $\left(\mathrm{M}^{\mathrm{pro}}\right)$ and papain-like protease $\left(\mathrm{PL}^{\mathrm{pro}}\right)$, responsible for replicating protein's proteolytic processing, likewise eliminating viral replication (Ibrahim et al. 2020; Hosseini et al. 2021). The essential roles of ACE-2, $\mathrm{M}^{\text {pro, }}$, and $\mathrm{PL}^{\mathrm{pro}}$ have rendered them as potential targets for discovering effectual medication against SARS-CoV-2 using molecular docking analysis (Naidoo et al. 2020). Numerous docking research studies have been conducted using $\mathrm{M}^{\text {pro }}, \mathrm{PL}^{\text {pro }}$, and ACE-2 receptors to discover a natural compound to fight against SARS-CoV-2. Flavonoids such as rutin, isorhamnetin-3-O- $\beta-\mathrm{D}$, and calendoflaside from Calendula officinalis have exhibited a superlative binding energy to main protease of SARS-CoV-2 than the native ligand (N3) (Joshi et al. 2020). Novel lichen compounds have also been investigated and have manifested a strong affinity toward $\mathrm{M}^{\text {pro }}$ and thus, can be established to be effective antiviral against SARS-CoV-2 ${ }^{1}$ (Joshi et al. 2020) ${ }^{7}$. Research has validated the combination of $\mathrm{PL}^{\text {pro }}$ and $\mathrm{M}^{\text {pro }}$ as an effective receptor in docking research to discover potential antiviral compounds from ebselene derivatives and cyanobacterial metabolites (Naidoo et al. 2020; Zmudzinski et al. 2020). Virtual screening focusing on ACE-2 
as the viral entry has been rigorously studied for several compounds and gave selected compounds to confirm an in vitro assay (Benítez-Cardoza and Vique-Sánchez 2020).

Molecular docking is used as a virtual screening to determine potential inhibitor candidates at several critical receptors in controlling the replication of SARS-CoV-2. Besides, pharmacochemical analyses, such as Absorption, Distribution, Metabolism and Excretion (ADME) analysis and toxicity prediction, are also used to complement molecular docking analysis recommendations. This research uses AutoDock Vina with a Lamarckian genetic algorithm. This software can optimize the interaction determination of potential candidate compounds. The ongoing COVID pandemic has necessitated an extensive research on the selection of the candidates found in active compounds of Indonesian biodiversity, both from organisms on land and sea with various bioactivity spectrums, that can effectively inhibit SARS-CoV-2 replication. This study strategically selected 102 potentially active compounds with target tethering to the ACE- $2, \mathrm{PM}^{\text {pro }}$, and $\mathrm{PL}^{\text {pro }}$ receptors. The potential was appraised in terms of the Gibbs free energy generated in the interaction, orientation, conformation, ADME analysis, and the associated toxicity analysis. This study is crucial as a recommendation for potential active compounds in identifying the effectual inhibitors of SARS-CoV-2 replication.

\section{Materials and methods}

\section{Software and hardware}

The whole research is computational using software and hardware involved in compound preparation, molecular docking, and analysis. We used the PubChem database for the compound preparation step for ligand molecules and Protein Data Bank (PDB) database for proteins. The preparation stage also removes water molecules, adds hydrogen atoms, and determines the receptor's ligand docking location using the AutoDockTools (ADT) software-1.5.6. Tethering molecules was done using AutoDock Vina, and the results were visualized using Discovery Studio 2020 and PyMol (Trott et al. 2010).

\section{Receptor preparation}

In this study, three receptors were used as ligand-tethered protein molecules, and the three receptors were ACE-2, $\mathrm{M}^{\text {pro }}$, and $\mathrm{PL}^{\text {pro }}$. The crystal structure of SARS-CoV-2 PL ${ }^{\text {pro }}$ (PDB ID: 5TL6) was downloaded via the website https:// www.rcsb.org/structure/5TL6 (Fig. 2) (Daczkowski et al. 2017). The crystal structure has a resolution of $2.62 \AA$, which is over 319 amino acids. The crystal structure binds $\mathrm{SO}_{4}$ ions and $\mathrm{Zn}$ ions. The SARS-CoV-2 main protease crystal structure (PDB ID: 6LU7) was downloaded via the website https://www.rcsb.org/structure/6LU7 (Fig. 1), with a crystal resolution of $2.16 \AA$ and composed of 306 amino acids. The 6LU7 crystal structure is accompanied by the ligand N3 (N - [(5-methylisoxazol-3-yl) carbonyl] alanyl-1-valyl-n $~ 1 \sim-((1 \mathrm{r}, 2 \mathrm{z})$-4- (benzyloxy) -4-oxo1 - ([((3r) -2-oxopyrrolidin-3-yl $]$ methyl $\}$ but-2-enyl) -L-leucinamide) (Jin et al. 2020). The crystal structure of ACE-2 (PDB ID: 1R42), bound to the NAG ligand (2-acetamido-2-deoxy-beta-D-glucopyranose), $\mathrm{Cl}$, and $\mathrm{Zn}$ ion (Towler et al. 2004). All the receptors were analyzed for their stability with a Ramachandran plot (Fig. 3). ADT 1.5.6 was used to remove water molecules, ligands, and natural ions and locate the receptor grid box's position. The results of the receptor preparations that are ready for molecular docking simulations were stored in the ${ }^{\star} \mathrm{PDB}$ QT format. The amino acids that affect the binding site of each receptor were presented in Table 3.

\section{Ligand preparation}

All chemical structures of natural materials from marine and terrestrial were obtained from PubChem (PubChem (nih.gov)) based on a thorough literature review. This study used several control compounds in the form of active compounds that were reported to have been used in the treatment of SARS-CoV-2 (Table 1).

MarvinSketch 20.12 was used to optimize each ligand's $2 \mathrm{D}$ and $3 \mathrm{D}$ structures and the conversion from ${ }^{*}$ mol files to ${ }^{\star} \mathrm{PDB}$ and ${ }^{\star} \mathrm{PDBQT}$. PDBQT file types were generally used for molecular docking simulations. The ligands used were also analyzed for toxicity and solubility using the Lipinski Rule (Lipinski et al. 2004), toxicity analysis using ADME (Cheng et al. 2012).

\section{Ligand-receptor docking}

Preparation of tethering between ligands and receptors was done using ADT 1.5.6 and AutoDock Vina 4.2 programs. The grid box's determination is one of the parameters used as the ligand docking region at the receptor. This study used 105 ligand-binding poses to the receptor by applying the Lamarckian Genetic Algorithm (LGA) to explore the ligand conformation (Morris et al. 1998). Molecular docking can assist in virtual screening by producing binding affinity energy $(\Delta \mathrm{G})$. The ligand with the maximum potential has a more negative value of $\Delta \mathrm{G}$. Discovery Studio was used to visualize the molecular docking between ligands and

\section{Results and discussion}

The active compounds of most of the Indonesian marine and terrestrial organisms used in this study have potential pharmaceutical applications. Table 1 encapsulates different classes of compounds that have been discovered, such as terpenoids, lipids, peptides, alkaloids, fatty acids, lactones, dioxins, xanthone, flavonoid, and phenols. Both terrestrial and marine organisms can produce immunomodulator and antiviral activities (i.e., against $\mathrm{HCV}, \mathrm{HBV}$, HSV, HIV, TMV, ISA, SINV, HCMC, JEV, and EV-71). 
Table 1. Compound type of Marine and Terrestrial Organisms.

\begin{tabular}{|c|c|c|c|c|c|}
\hline No. & Chemical Structure & Compound & Type & Organism & Ref. \\
\hline 1. & & Eurycomanone & Alkaloid & Eurycoma longifolia & Sitanggang et al. 2018 \\
\hline 2. & & Isovitexin & Flavonoid & Artemisia lactiflora & Wang et al. 2020 \\
\hline 3. & & Lupeol & Flavonoid & Moringa oleifera & Malinowska et al. 2015 \\
\hline 4. & & Methyl 3, 5-di-O-caffeoyl quinate & Polyphenol & Artemisia lactiflora & Hung et al. 2006 \\
\hline 5. & & Neoandrographolide & Flavonoid & Andrographis paniculate & $\begin{array}{l}\text { Liu et al. 2007; Kamden } \\
\text { et al. } 2007\end{array}$ \\
\hline 6. & & Proanthocyanidins & Polyphenol & Сіпnатотит burmanii & Plumb et al. 1998 \\
\hline 7. & & (-) Alphapinene & Polyphenol & Kaempferia galangal L. & $\begin{array}{l}\text { Yang et al. 2010; Jiang et } \\
\text { al. 2011 }\end{array}$ \\
\hline 8. & & (+) Alphapinene & Polyphenol & Kaempferia galangal L. & $\begin{array}{l}\text { Yang et al. 2010; Jiang et } \\
\text { al. 2011 }\end{array}$ \\
\hline 9. & $\mathrm{O}$ & (E)_cinnamaldehyde & Phenol & Сіпnатотит burmanii & Chen et al. 2017 \\
\hline
\end{tabular}




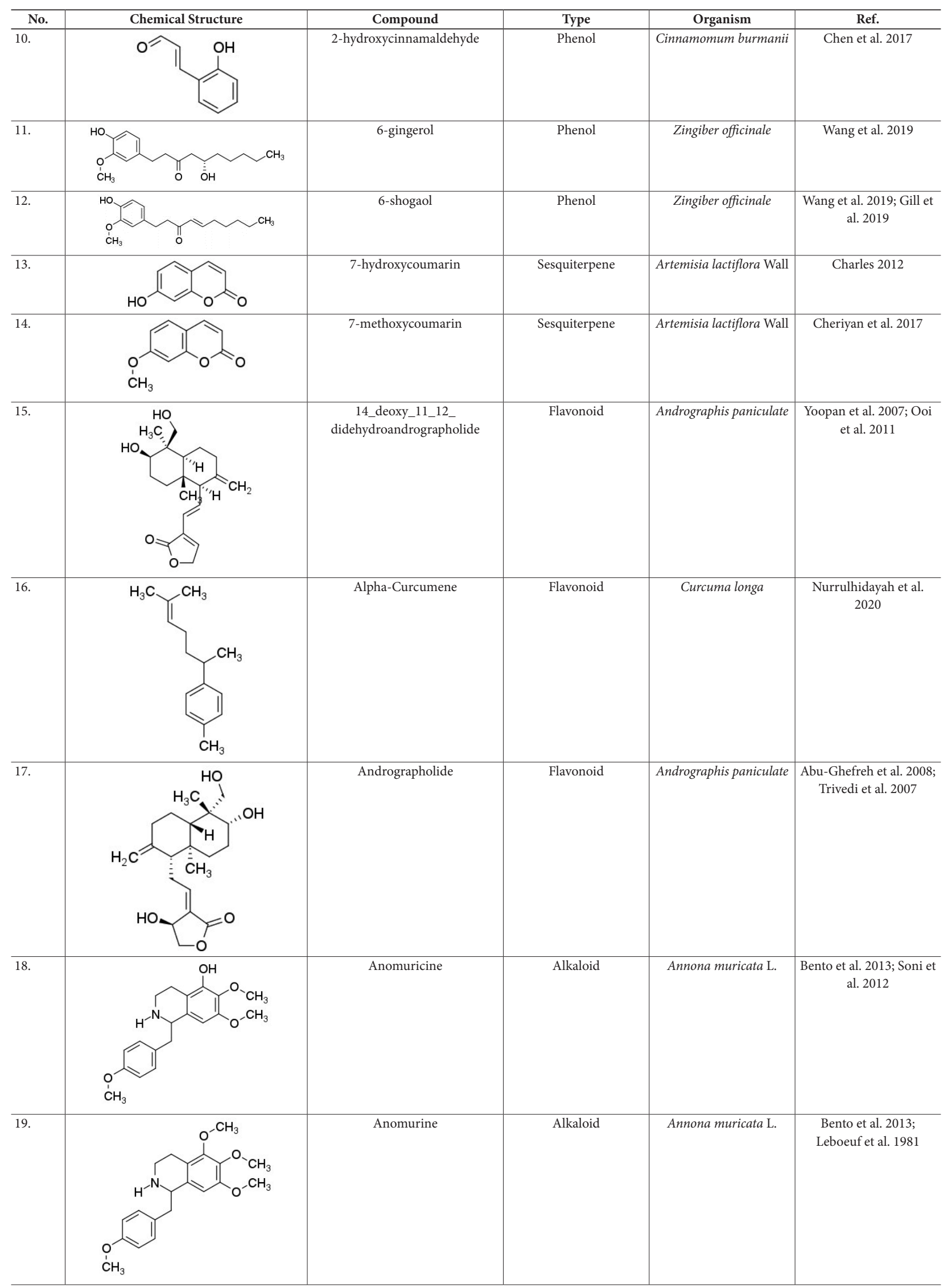




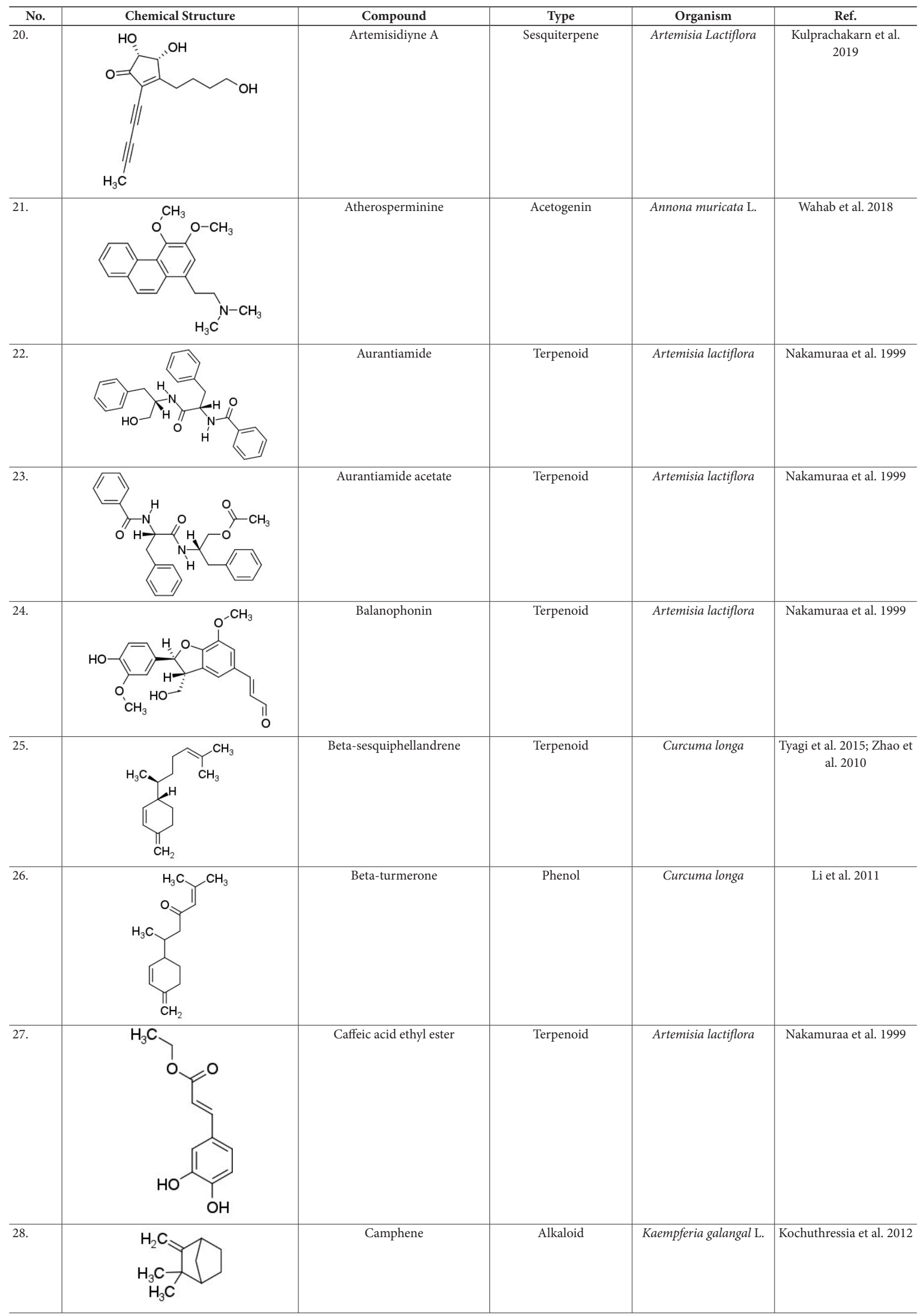




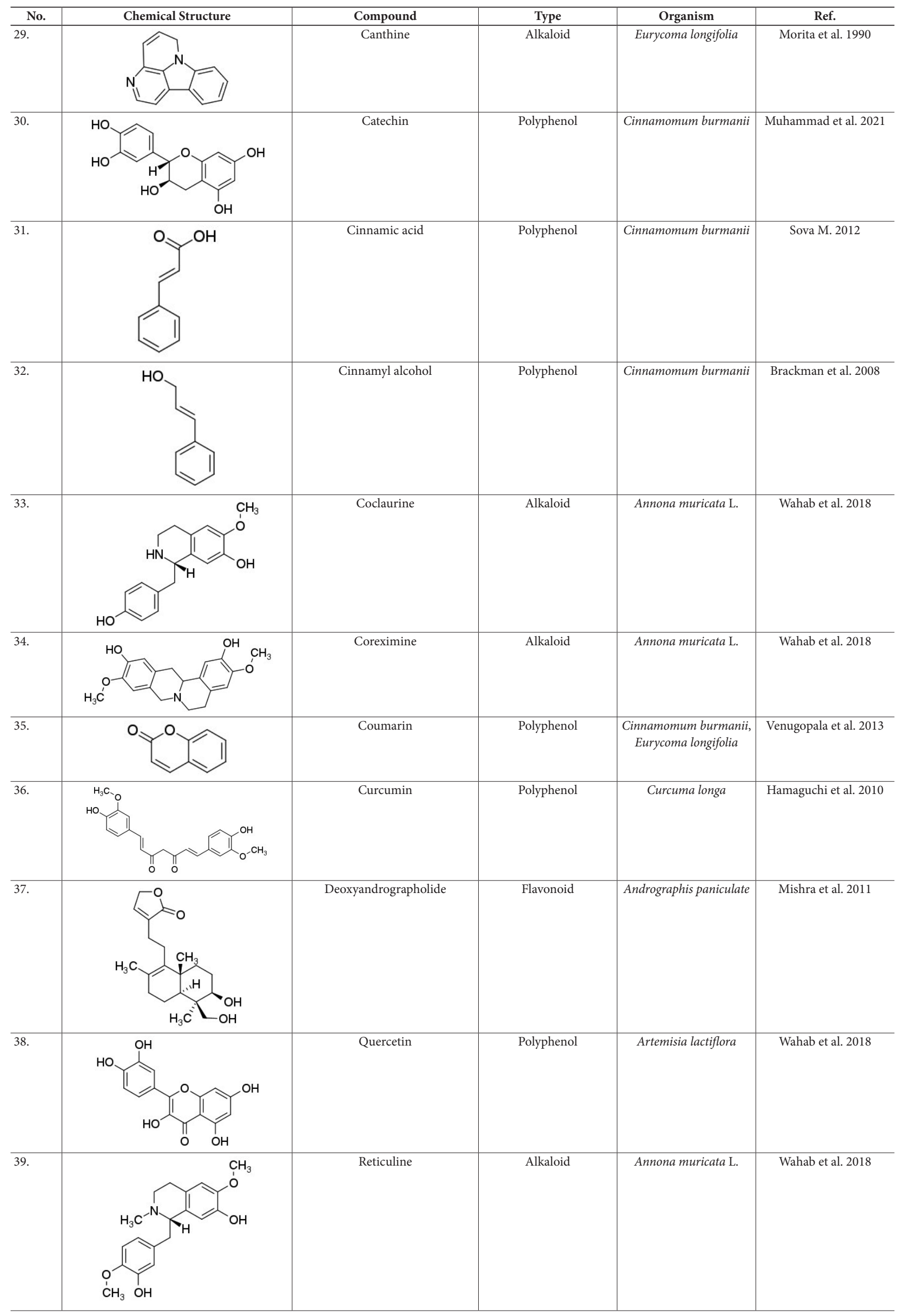




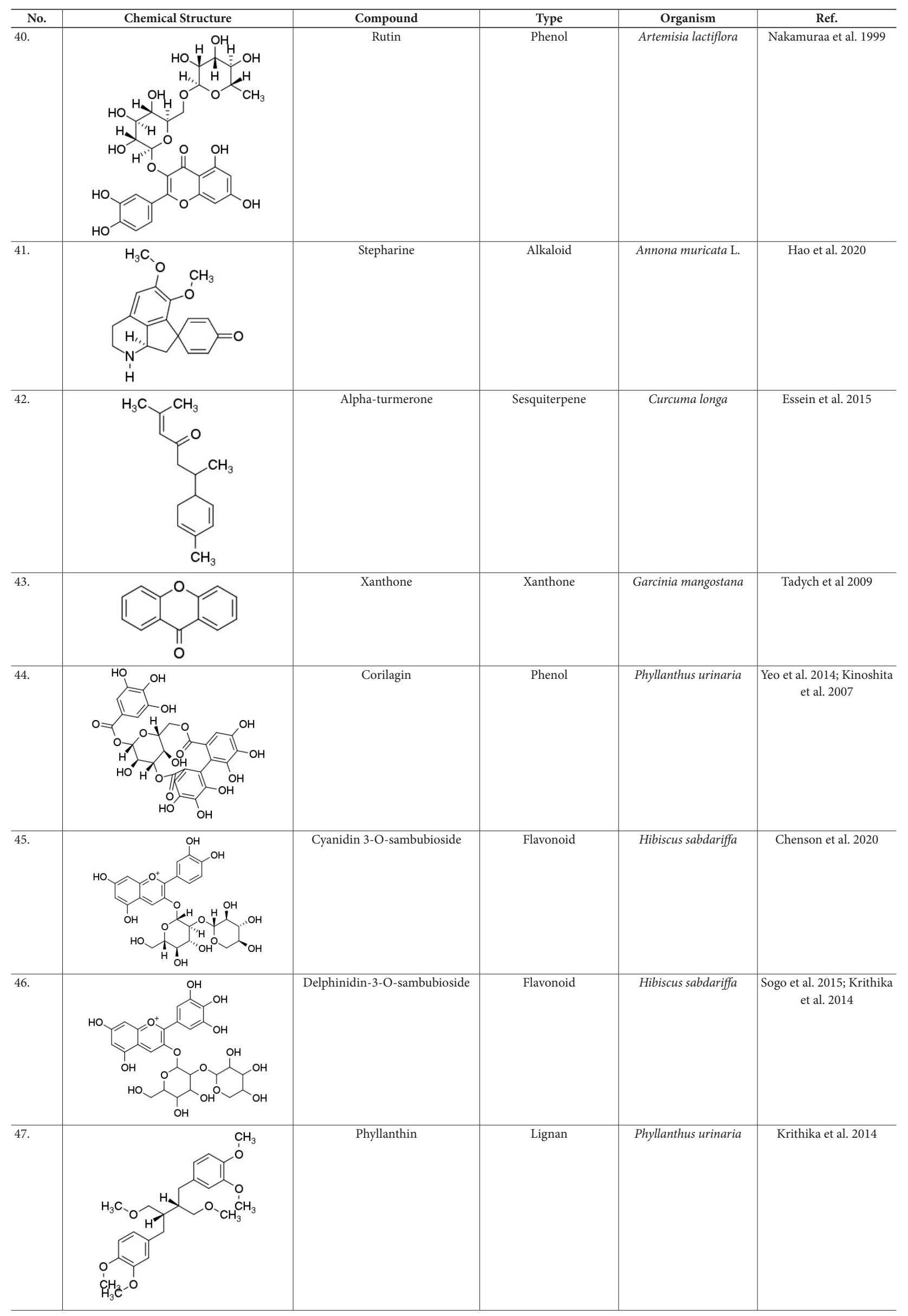




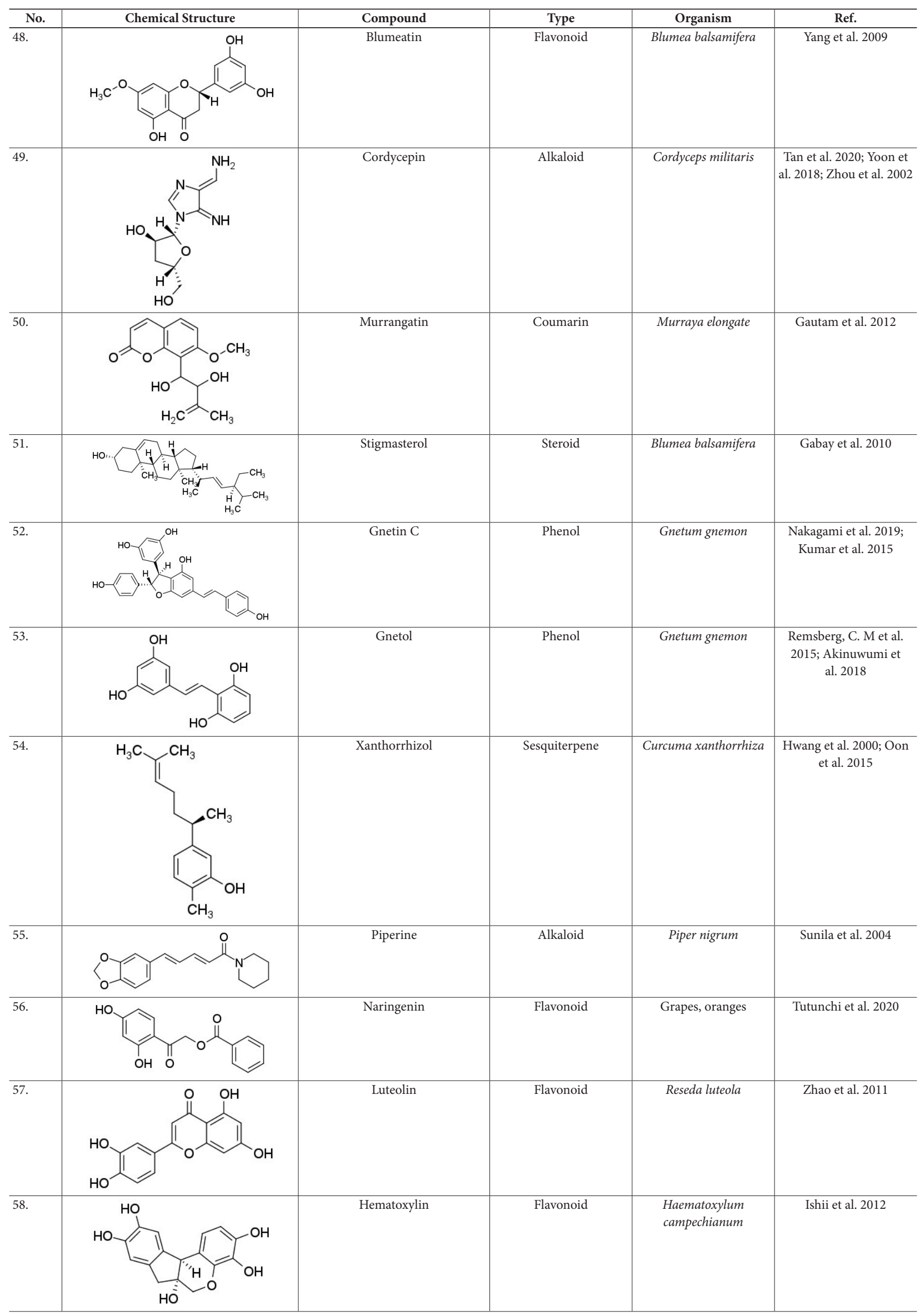




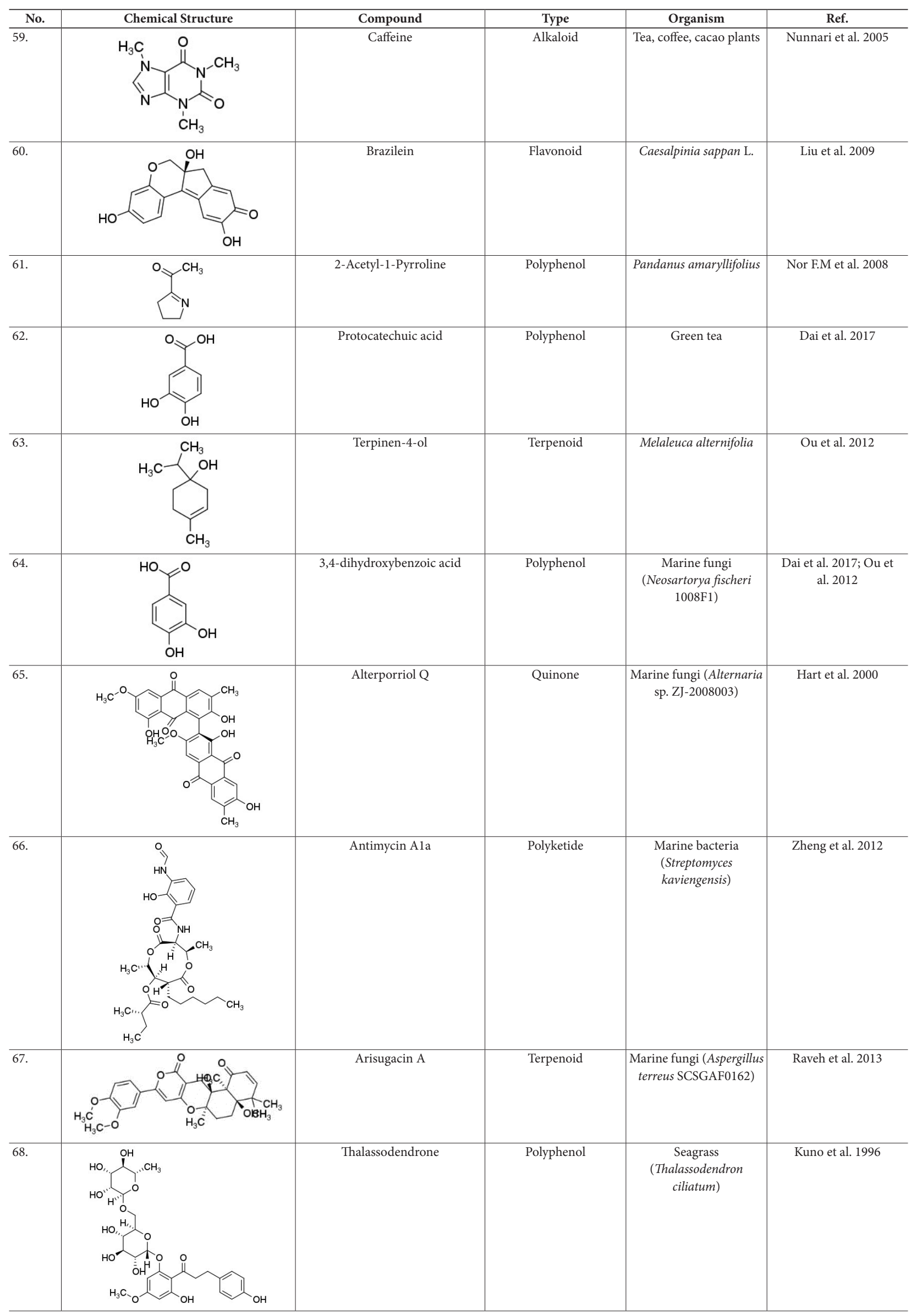




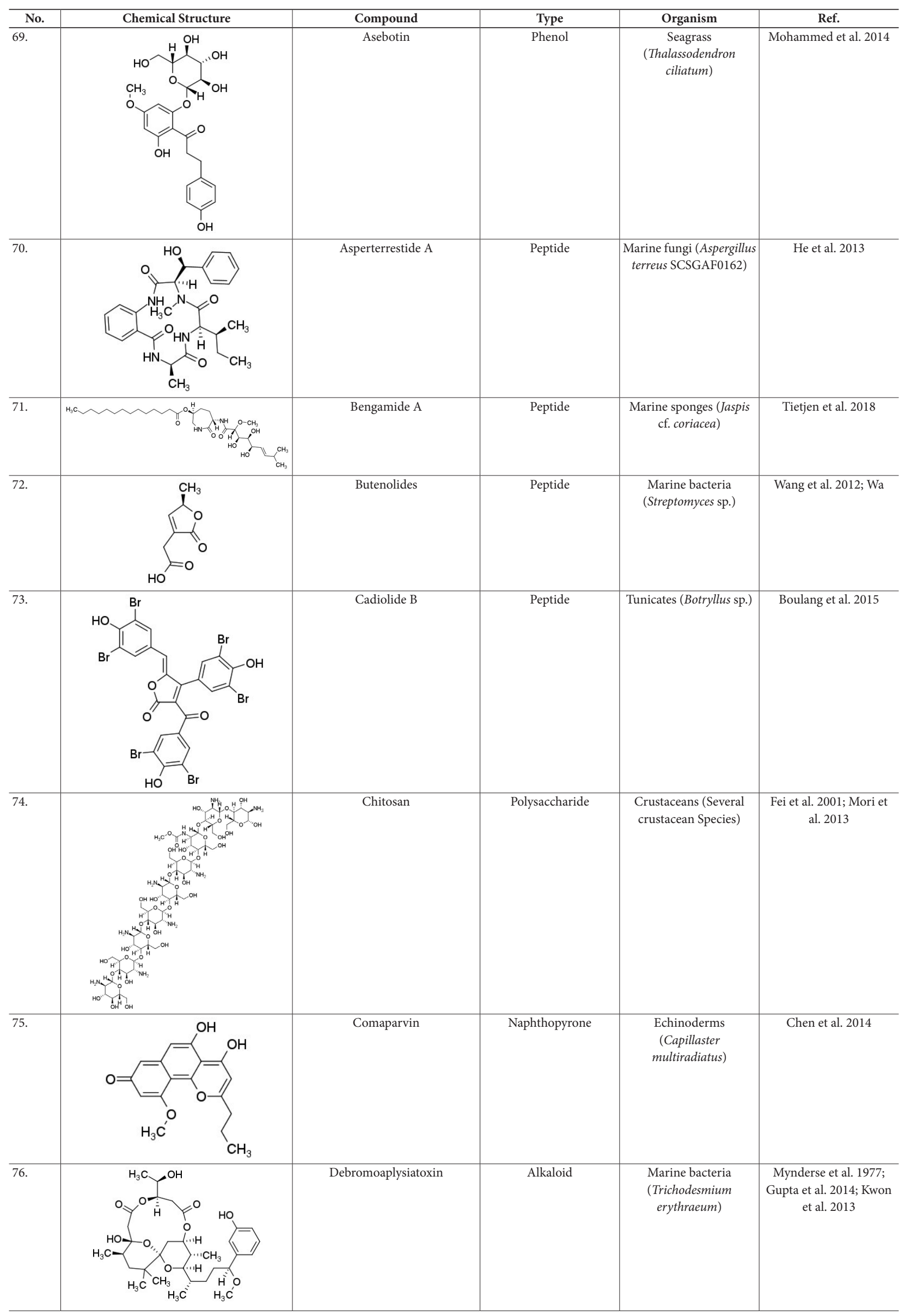




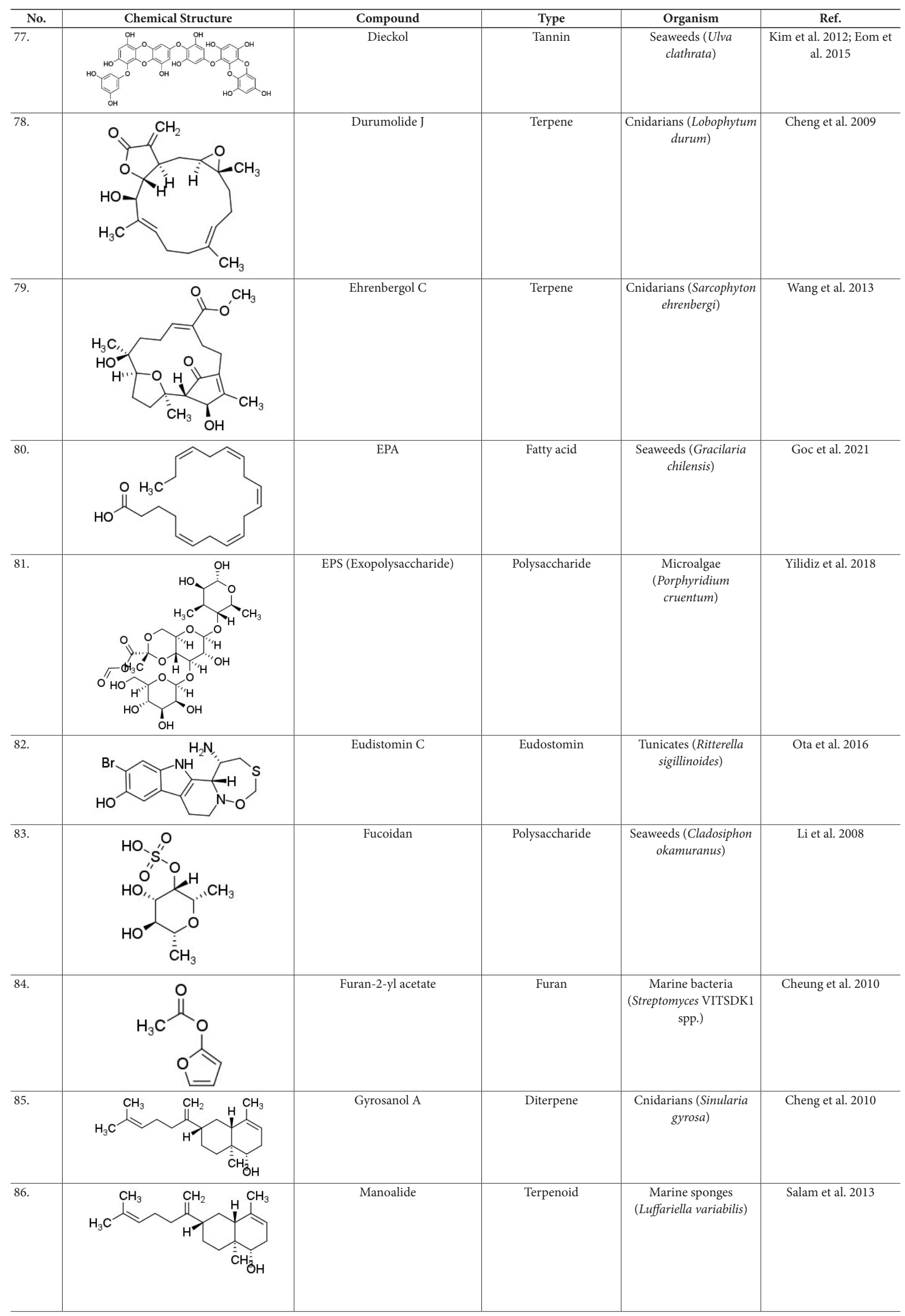




\begin{tabular}{|c|c|c|c|c|c|}
\hline No. & Chemical Structure & Compound & Type & Organism & Ref. \\
\hline 87. & & Metachromin A & Terpenoid & $\begin{array}{c}\text { Marine sponges } \\
\text { (Dactylospongia } \\
\text { metachromia) }\end{array}$ & Yamashita et al. 2017 \\
\hline 88. & & MGDG & Lipid & $\begin{array}{c}\text { Microalgae (Coccomyxa } \\
\text { sp. KJ) }\end{array}$ & Hayashi et al. 2019 \\
\hline 89. & & Mollamide F & Peptide & $\begin{array}{c}\text { Tunicates (Didemnum } \\
\text { molle) }\end{array}$ & Lu et al. 2012 \\
\hline 90. & & Molleurea A & Benzene derivative & $\begin{array}{l}\text { Tunicates (Didemnum } \\
\text { molle) }\end{array}$ & Ji et al. 2018 \\
\hline 91. & & Nortopsentins & Alkaloid & $\begin{array}{c}\text { Marine sponges } \\
\text { (Spongosorites ruetzleri) }\end{array}$ & Lozano et al. 2016 \\
\hline 92. & & Omega-3 & Fatty acid & $\begin{array}{c}\text { Seaweeds (Gracilaria } \\
\text { chilensis) }\end{array}$ & $\begin{array}{l}\text { Gonzalez-Almela et al. } \\
2015\end{array}$ \\
\hline 93. & & Pateamine A & Lactone & $\begin{array}{l}\text { Marine sponges } \\
\text { (Mycale sp.) }\end{array}$ & Ryu et al. 2011 \\
\hline 94. & & Phlorofucofuroeckol A & Dioxin & $\begin{array}{l}\text { Seaweeds (Ulva } \\
\text { clathrata) }\end{array}$ & Cheng et al. 2014 \\
\hline 95. & & Secocembranoid & Diterpene & $\begin{array}{c}\text { Cnidarians (Lobophytum } \\
\text { crassum) }\end{array}$ & Smitha et al. 2014 \\
\hline 96. & & Prunolide A & Hydrocarbon cyclic & $\begin{array}{c}\text { Tunicates (Synoicum } \\
\text { prunum) }\end{array}$ & Qin et al. 2015 \\
\hline
\end{tabular}




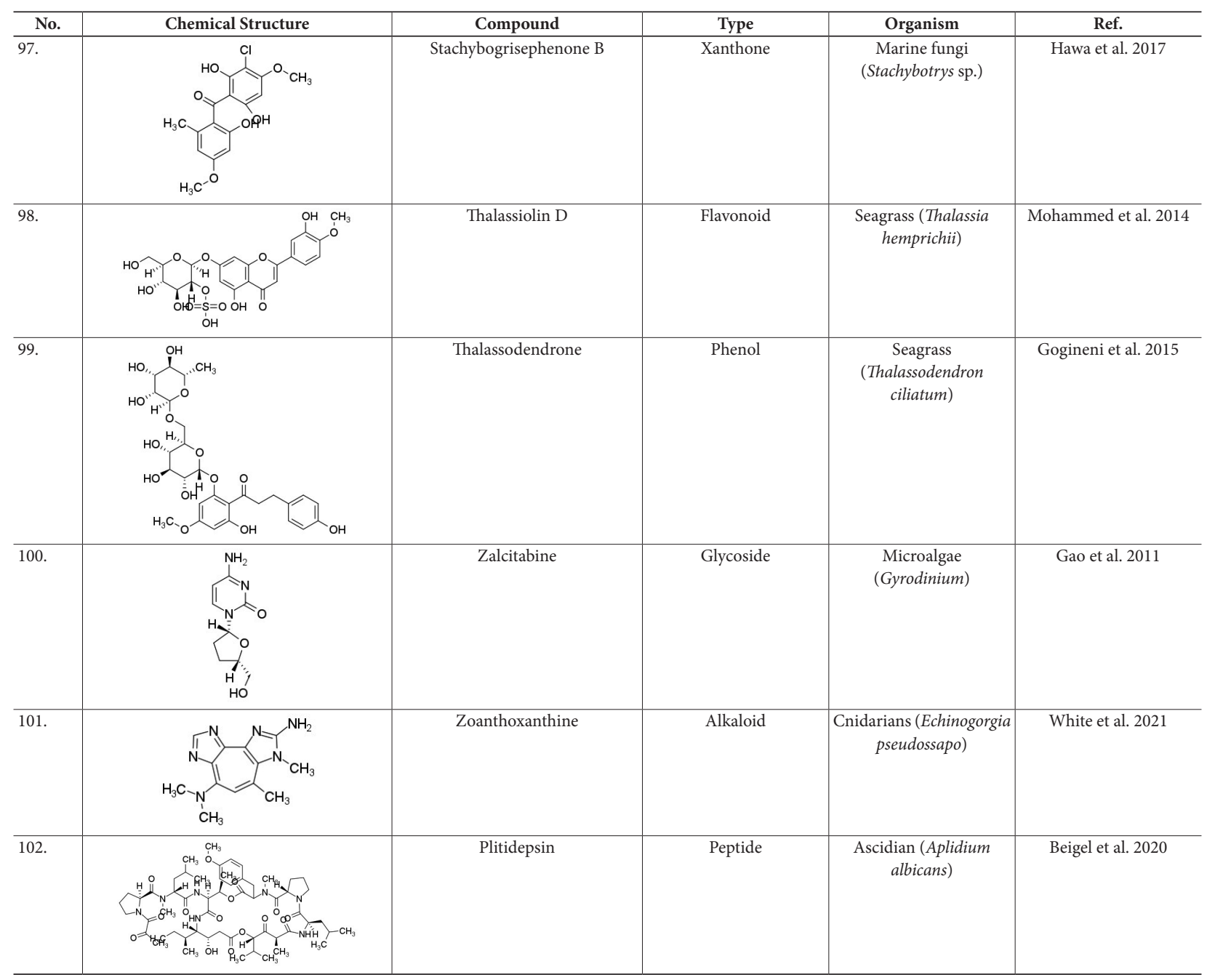

The usefulness of species of Indonesian terrestrial organisms in this study was based on a thorough literature study. Most researchers believe these plants can enrich health and prevent diseases caused by the COVID-19 pandemic even though most of these plants have not been validated as effectual against COVID-19 heretofore. We use marine bacteria, fungi, microalgae, marine plants, and marine invertebrate for marine compounds.

Several studies have affirmed that terrestrial and marine organisms produce various compounds derived from secondary metabolism, requiring antiviral activity. More particularly, certain terrestrial and marine metabolites are active against some viruses. Besides, various mechanisms and different targets of action have been discovered. Mechanisms of action of possible antiviral compounds are diversified because they can block viruses at different stages of their life cycles. Typical viral life cycle stages are attachment, penetration, uncoating, replication, assembly, and release (Ibrahim et al. 2020). We aimed to procure information through molecular docking regarding plants and marine compounds that have the antiviral activity to be used against SARS-CoV-2.

As a comparative study, we also used common drugs, namely Remdesivir, Chloroquine, and Favipiravir, which have been used as common drugs for specific diseases and can be used to expedite the treatment process of SARS-CoV-2. For instance, Favipiravir is one such oral drug approved for a new and resurfacing influenza pandemic in Japan in 2014 and has exhibited potent in vitro activity against SARS-CoV-2 (Hosseini et al. 2021). We also use Plitidepsin, originally from Ascidian, approved for drug marketing and reported to have potent preclinical efficacy against SARSCoV-2 by targeting the host protein eEF1A (Lan et al. 2020).

The analysis on the structure-activity relationship understanding is essential for further study; for example, analysis on the structure-activity relationship revealed that the hydroquinone moiety and the double bonds at carbon numbers-5 and -9 in metachromatic A are crucial for anti-HBV activity (Sherren et al. 2020). Another example MGDG could cause complete lysis of the viral envelope, which is essential for viral attachment to host cells. This phenomenon likely explains the virucidal action of MGDG (Liu et al. 2020).

\section{Receptor structure and stability}

SARS-CoV-2 Mpro receptor with a PDB ID of 6LU7 has a resolution of $2.16 \AA$ with 306 amino acids. The $\mathrm{M}^{\text {pro }} \mathrm{SARS}$ - 
Table 2. ADME and Lipinski's analyses from secondary metabolites.

\begin{tabular}{|c|c|c|c|c|c|c|c|c|c|c|c|}
\hline \multicolumn{12}{|c|}{ ADME and Lipinski analyses } \\
\hline Compound & MW (g/mol) & $\log P$ & HBD & HBA & Lipinski & Compounds & MW (g/mol) & $\log P$ & HBD & HBA & Lipinski \\
\hline Eurycomanone & 408.4 & -0.89 & 5 & 9 & Yes & Alterporriol Q & 566.51 & 0.29 & 4 & 10 & Yes \\
\hline Isovitexin & 432.38 & -2.02 & 7 & 10 & Yes & Antimycin Ala & 548.63 & 2.18 & 3 & 9 & No \\
\hline Lupeol & 426.72 & 6.92 & 1 & 1 & Yes & Arisugacin A & 496.55 & 1.57 & 2 & 8 & Yes \\
\hline Methyl 3, 5-di-O-caffeoyl quinate & 530.48 & -0.15 & 6 & 12 & No & Asebogenin & 288.3 & 1.35 & 3 & 5 & Yes \\
\hline Neoandrographolide & 480.59 & 1.26 & 4 & 8 & Yes & Asebotin & 450.44 & -1.2 & 6 & 10 & Yes \\
\hline Proanthocyanidins & 592.55 & -0.07 & 9 & 12 & No & Asperterrestide A & 480.56 & 0.86 & 4 & 5 & Yes \\
\hline (-) Alphapinene & 136.23 & 4.29 & 0 & 0 & Yes & Bengamide A & 584.78 & 1.01 & 5 & 8 & Yes \\
\hline (+) Alphapinene & 136.23 & 4.29 & 0 & 0 & Yes & Butenolides & 156.14 & 0.11 & 1 & 4 & Yes \\
\hline (E)_cinnamaldehyde & 132.16 & 2.01 & 0 & 1 & Yes & Cadiolide B & 873.76 & 5.43 & 3 & 6 & No \\
\hline 2-hydroxycinnamaldehyde & 148.16 & 1.35 & 1 & 2 & Yes & Chitosan & 1526.45 & -17.69 & 29 & 47 & No \\
\hline 6-gingerol & 294.39 & 2.14 & 2 & 4 & Yes & Comaparvin & 300.31 & 1.05 & 2 & 5 & Yes \\
\hline 6-shogaol & 276.37 & 2.9 & 1 & 3 & Yes & Debromoaplysiatoxin & 592.72 & 2.19 & 3 & 10 & Yes \\
\hline 7-hydroxycoumarin & 162.14 & 1.04 & 1 & 3 & Yes & Dieckol & 742.55 & 0.04 & 11 & 18 & No \\
\hline 7-methoxycoumarin & 176.17 & 1.34 & 0 & 3 & Yes & Durumolide J & 332.43 & 2.72 & 1 & 4 & Yes \\
\hline $\begin{array}{l}\text { 14_deoxy_11_12_ } \\
\text { didehydroandrographolide }\end{array}$ & 332.43 & 2.72 & 2 & 4 & Yes & Ehrenbergol C & 316.43 & 3.48 & 1 & 3 & Yes \\
\hline Alpha-curcumene & 202.34 & 5.75 & 0 & 0 & Yes & EPA & 302.45 & 4.67 & 1 & 2 & Yes \\
\hline Andrographolide & 350.45 & 1.98 & 3 & 5 & Yes & EPS (Exopolysaccharide) & 600.52 & -4.31 & 8 & 18 & No \\
\hline Anomuricine & 329.39 & 1.75 & 2 & 5 & Yes & Eudistomin C & 370.26 & 1.54 & 3 & 4 & Yes \\
\hline Anomurine & 343.42 & 1.98 & 1 & 5 & Yes & Fucoidan & 242.25 & -1.83 & 3 & 7 & Yes \\
\hline Artemisidiyne A & 248.27 & 0.46 & 3 & 4 & Yes & Furan-2-yl acetate & 126.11 & 0.42 & 0 & 3 & Yes \\
\hline Atherosperminine & 309.4 & 3.36 & 0 & 3 & Yes & Gyrosanol A & 288.47 & 4.65 & 1 & 1 & Yes \\
\hline Aurantiamide & 402.49 & 3.07 & 3 & 3 & Yes & Manoalide & 416.55 & 3.69 & 2 & 5 & Yes \\
\hline Aurantiamide acetate & 444.52 & 3.41 & 2 & 4 & Yes & Metachromin A & 358.47 & 2.18 & 1 & 4 & Yes \\
\hline Balanophonin & 356.37 & 1.01 & 2 & 6 & Yes & MGDG & 688.97 & 2.87 & 4 & 10 & Yes \\
\hline Beta-sesquiphellandrene & 204.35 & 4.53 & 0 & 0 & Yes & Mollamide F & 638.82 & 0.62 & 3 & 6 & No \\
\hline Beta-turmerone & 218.33 & 3.37 & 0 & 1 & Yes & Molleurea A & 415.53 & 4.09 & 3 & 2 & Yes \\
\hline Caffeic acid ethyl ester & 208.21 & 1.3 & 2 & 4 & Yes & Nortopsentin & 298.34 & 2.23 & 3 & 1 & Yes \\
\hline Camphene & 136.23 & 4.29 & 0 & 0 & Yes & Omega-3 & 304.47 & 4.75 & 1 & 2 & Yes \\
\hline Canthine & 206.24 & 2.36 & 0 & 1 & Yes & Pateamine A & 555.77 & 2.91 & 1 & 7 & Yes \\
\hline Catechin & 290.27 & 0.24 & 5 & 6 & Yes & Phlorofucofuroeckol A & 602.46 & 0.35 & 9 & 14 & No \\
\hline Cinnamic acid & 148.16 & 1.9 & 1 & 2 & Yes & Secocembranoid & 304.47 & 3.94 & 0 & 2 & Yes \\
\hline Cinnamyl alcohol & 118.18 & 4.08 & 0 & 0 & Yes & Prunolide A & 1205.7 & 7.46 & 4 & 9 & No \\
\hline Coclaurine & 285.34 & 1.84 & 3 & 4 & Yes & Stachybogrisephenone B & 338.74 & 1.32 & 3 & 6 & Yes \\
\hline Coreximine & 327.37 & 1.75 & 2 & 5 & Yes & Thalassiolin D & 542.47 & -2.25 & 6 & 14 & No \\
\hline Coumarin & 146.14 & 1.65 & 0 & 2 & Yes & Thalassodendrone & 596.58 & -2.57 & 8 & 14 & No \\
\hline Curcumin & 368.38 & 1.47 & 2 & 6 & Yes & Zalcitabine & 211.22 & -0.7 & 2 & 4 & Yes \\
\hline Deoxyandrographolide & 334.45 & 2.81 & 2 & 4 & Yes & Zoanthoxanthine & 256.31 & 1.09 & 1 & 3 & Yes \\
\hline Quercetin & 302.24 & -0.56 & 5 & 7 & Yes & 2-Acetyl-1-Pyrroline & 111.14 & -0.04 & 0 & 2 & Yes \\
\hline Reticuline & 329.39 & 1.75 & 2 & 5 & Yes & Brazilein & 284.26 & 0.42 & 3 & 5 & Yes \\
\hline Rutin & 610.52 & -3.89 & 10 & 16 & No & Caffeine & 194.19 & 0.22 & 0 & 3 & Yes \\
\hline Stepharine & 297.35 & 1.81 & 1 & 4 & Yes & Chloroquine & 319.87 & 3.2 & 1 & 2 & Yes \\
\hline Alpha-turmerone & 218.33 & 3.37 & 0 & 1 & Yes & Favipiravir & 157.1 & -1.3 & 2 & 4 & Yes \\
\hline Xanthone & 196.2 & 2.06 & 0 & 2 & Yes & Gnetin C & 454.47 & 2.86 & 5 & 6 & Yes \\
\hline Corilagin & 634.45 & -2.42 & 11 & 18 & No & Gnetol & 244.24 & 1.67 & 4 & 4 & Yes \\
\hline Cyanidin 3-O-sambubioside & 581.5 & -3.28 & 10 & 15 & No & Hematoxyline & 302.28 & 0.49 & 5 & 6 & Yes \\
\hline Delphinidin-3-O-sambubioside & 597.5 & -3.75 & 11 & 16 & No & Luteolin & 286.24 & -0.03 & 4 & 6 & Yes \\
\hline Phyllanthin & 418.52 & 2.43 & 0 & 6 & Yes & Murrangatin & 276.28 & 0.95 & 2 & 5 & Yes \\
\hline Blumeatin & 302.28 & 0.41 & 3 & 6 & Yes & Naringenin & 272.25 & 0.71 & 3 & 5 & Yes \\
\hline Cordycepin & 251.24 & -1.94 & 3 & 6 & Yes & Piperine & 285.34 & 2.39 & 0 & 3 & Yes \\
\hline Plitidepsin & 1110.34 & -1.12 & 4 & 15 & No & Protocatechuic acid & 154.12 & 0.4 & 3 & 4 & Yes \\
\hline Remdesivir & 602.58 & 0.18 & 4 & 12 & No & Terpinen-4-ol & 154.25 & 2.3 & 1 & 1 & Yes \\
\hline Stigmasterol & 412.69 & 6.62 & 1 & 1 & Yes & Xanthorrhizol & 218.33 & 4.03 & 1 & 1 & Yes \\
\hline 3,4-dihydroxybenzoic acid & 154.12 & 0.4 & 3 & 4 & Yes & & & & & & \\
\hline
\end{tabular}

CoV-2 protein structure consists of $10 \alpha$-helix structures at the positions $\alpha 1$ : residue $10-15, \alpha 2$ : residue $41-44, \alpha 3$ : residue: 53-60, a4: residue 62-66, a5: residue 200-214, a6: residue: 226-237, a7: residue 243-250, a8: residue 250-258, a9: residue 260-275, and a10: residue: 292-301. In addition to the helix structure, the $\mathrm{M}^{\text {pro }}$ protein was also composed of $13 \beta$-sheet structures at positions $\beta 1$ : residue $17-22, \beta 2$ : residue $25-32, \beta 3$ : residue $35-29, \beta 4$ : residue $67-70, \beta 5$ : residue $73-75$, $\beta 6$ : residue $77-83, \beta 7$ : residue $86-91, \beta 8$ : residue $101-103$, $\beta 9$ : residue $111-118$, $\beta 10$ : residue $121-129, \beta 11$ : residue $148-152, \beta 12$ : residue $157-166, \beta 13$ : residue $172-175$. In the $\beta$-sheet structure of $\mathrm{M}^{\text {pro }}$ protein, it was known that the $\beta 1-\beta 7$ position is an antiparallel $\beta$-sheet structure, while the $\beta 8-\beta 1-13$ position is a $\beta$-sheet structure with both parallel and antiparallel $\beta$-sheet structures. The remaining areas are loop or turn areas composed of amino acids connecting the alpha and beta structures. 

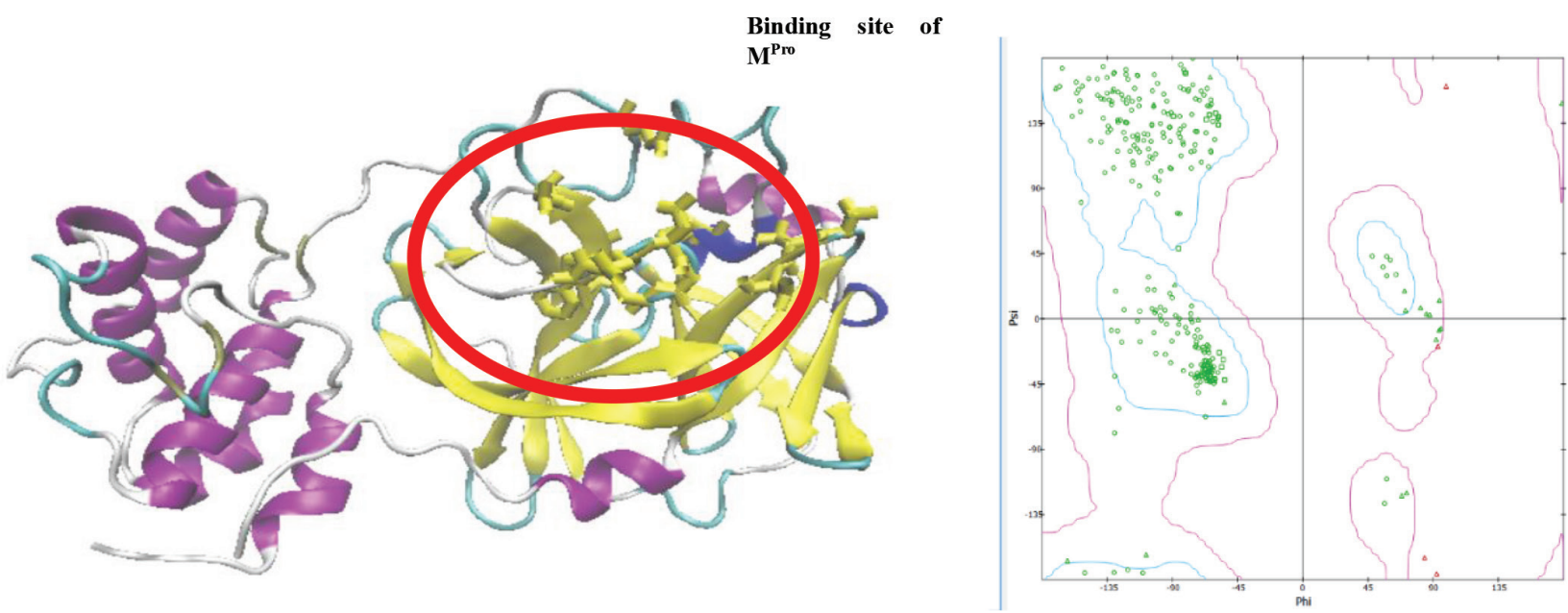

Figure 1. The structure of the $\mathrm{M}^{\text {pro }}$ SARS-CoV-2 receptor (PDB ID: 6LU7) along with the distribution of residues on the Ramachandran plot. The $\mathrm{M}^{\text {pro }}$ receptor structure is composed of $10 \alpha$-helix structures and $13 \beta$-sheet structures. The $\mathrm{M}^{\text {pro }}$ receptor structure is ready to use in molecular docking simulations with $92.15 \%$ of the residue in the protein-forming region.
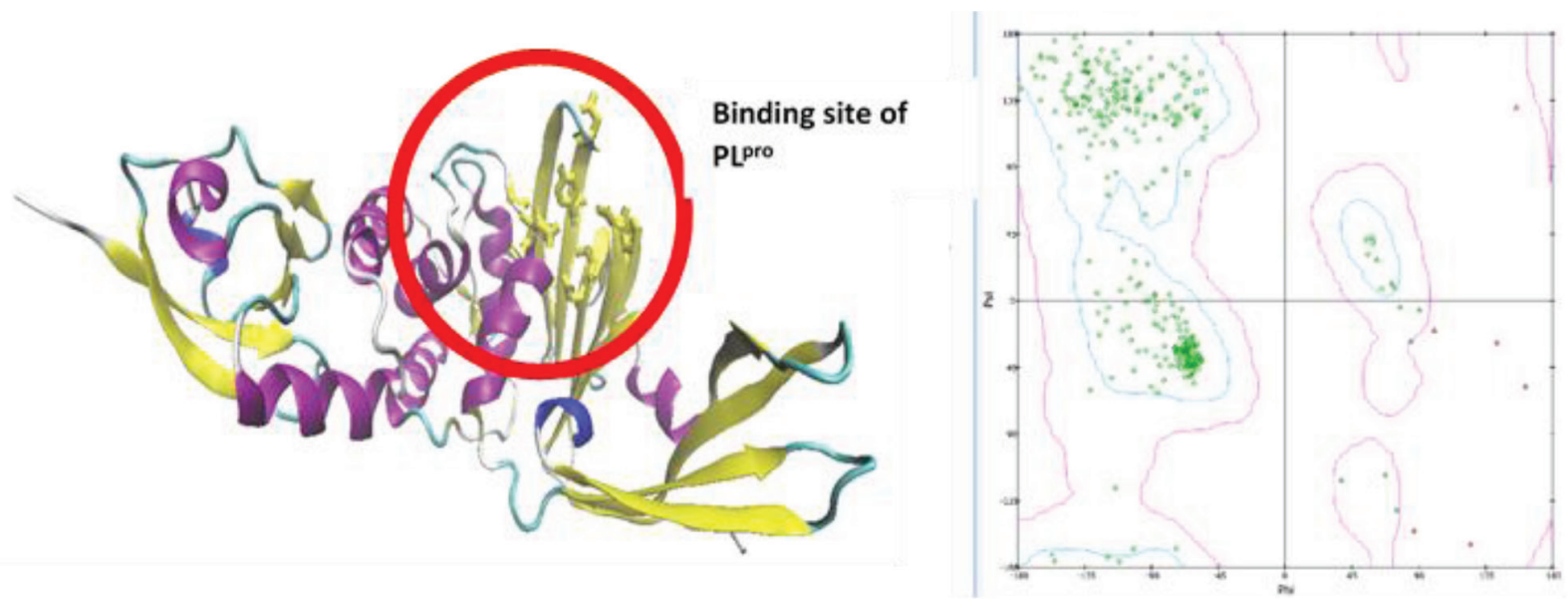

Figure 2. The structure of the PL pro SARS-CoV-2 receptor (PDB ID: 5TL6) along with the distribution of residues on the Ramachandran plot. The $\mathrm{PL}^{\text {pro }}$ receptor structure is composed of $10 \alpha$-helix structures and $19 \beta$-sheet structures. The PL ${ }^{\text {pro }}$ receptor structure is ready to use in molecular docking simulations with $96.55 \%$ of the residue in the protein-forming region.

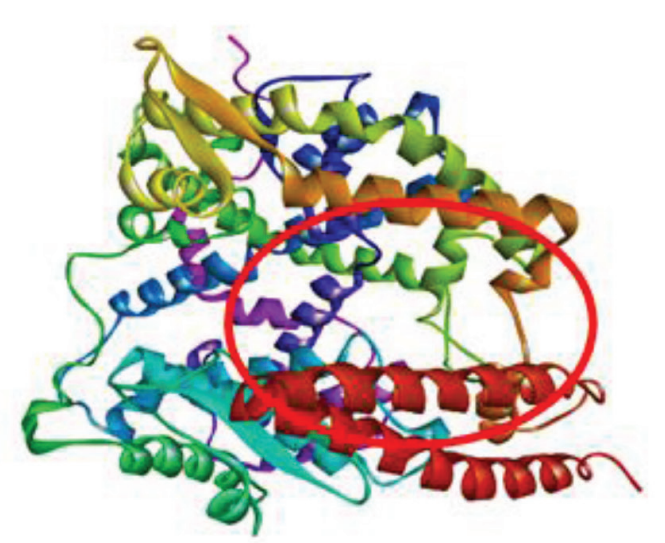

Binding site of ACE2

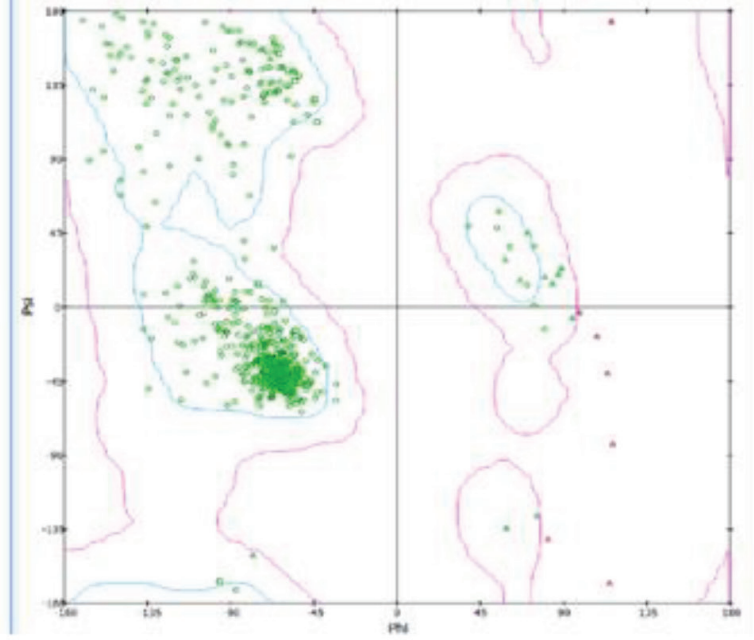

Figure 3. Structure of the ACE 2 receptor h receptor (PDB ID: 1R42) along with the distribution of residues on the Ramachandran plot. The ACE- 2 receptor structure is composed of $31 \alpha$-helix structures and six $\beta$-sheet structures. The structure of the hACE- 2 receptor is ready to be used in molecular docking simulations with $98.37 \%$ of the residue in the protein-forming region. 
The $\mathrm{M}^{\text {pro }}$ receptor has been meticulously analyzed for its structure and stability using the Ramachandran's plot. Stable structures have a resolution of less than $2.5 \AA$ with amino acid residues in the protein-forming region above 85\% (Trott et al. 2010). The $\mathrm{M}^{\text {pro }} \mathrm{SARS}-\mathrm{CoV}-2$ receptor has a resolution below $2.5 \AA$ with the amino acid residue scattered in the region that makes up the protein structure is 92.15\% (282/306). The $\mathrm{M}^{\text {pro }}$ receptor has 14 residues that interact with ligands, namely Thr25, Thr25, Thr26, His41, Phe140, Leu141, Asn142, Gly143, Ser144, Cys145, His163, His164, Glu166, and Gln189. The grid box dimensions (x, y, z) $(\AA)$ are $(50,50,50)$, with centers $(-10.850,-15.320$, 68.390) (Daczkowski et al. 2017).

The structural analysis of the $\mathrm{PL}^{\text {pro }}$ receptor with a PDB ID 5 TL6 revealed that it consists of 319 amino acids having a resolution of $2.62 \AA$ with $96.55 \%$ (308/319) residues are in the protein-forming region. The $\mathrm{PL}^{\text {pro }}$ protein is composed of $10 \alpha$-helix structures and $19 \beta$-sheet structures. The $\alpha$-helix structure is at positions $\alpha 1$ : residue $30-36$, $\alpha 2$ : residue 51-55, a3: residue 65-77, a4: residue 82-95, a5: residue 114-125, a6: residue 133-144, a7: residue 148-159, a8: residue 168-178, a9: residue 205-209, a10: residue 213-220. Meanwhile, the $\beta$-sheet structure is at positions $\beta 1$ : residue $8-14, \beta 2$ : residue $21-26, \beta 3$ : residue $38-40, \beta 4$ : residue $43-44, \beta 5$ : residue $58-61, \beta 6$ : residue $101-102, \beta 7$ : residue $105-106, \beta 8$ : residue $186-192$, $\beta 9$ : residue 198 204, $\beta 10$ : residue $210-212, \beta 11$ : residue $224-227, \beta 12$ : residue $233-242, \beta 13$ : residue $245-257, \beta 14$ : $264-271$, $\beta 15$ : residue $274-282, \beta 16$ : residue $286-290, \beta 17$ : residue 293-297, $\beta 18$ : residue $300-310, \beta 19$ : residue $311-312$. The rest is a connecting structure including a loop and turn structure. Some residues with ligands are Gly166, Asp167, Pro250, Pro251, Tyr267, Asn270, Tyr271, Tyr276, Thr304, and Asp305 (Hosseini et al. 2021). The grid box dimensions $(\mathrm{x}, \mathrm{y}, \mathrm{z})(\AA)$ are $(40,50,40)$, with centers $(-7.615$, $-6.980,-35.360)$.

The human angiotensin converting enzyme (hACE-2) receptors with PDB ID 1R42 have been analyzed for structure and stability, with a resolution value of $2.2 \AA$ consisting of 615 amino acids with $98.37 \%(605 / 615)$ residues located in the protein structure-forming region. ACE-2 protein is dominated by $a$-helix structure, with a total of $31 \alpha$-helix structures and six $\beta$-sheet structures with positions a1: residue 22-53, $\alpha 2$ : residue 55-78, a3: residue 90-101, a4: residue 103-108, a5: residue 109-130, a6: residue 147-155, a7: residue 157-172, a8: residue 172-194, a9: residue 198-205, a10: residue 219-252, a11: residue 265-267, a12: residue 275-277, a13: residue 278-283, a14: residue 297-301, a15: residue 303-317, a16: residue 326-331, a17: residue 365-385, a18: residue 389-393, a19: residue 399-414, a20: residue 414-421, a21: residue 431-447, a22: residue 448-446, a23: residue 469-471, a24: residue 472-484, a25: residue 498-502, a26: residue 503-508, a27: residue 513-532, a28: residue 538-542, a29: residue 547-559, $\alpha 30$ : residue 565-575, a31: residue 581-599. Meanwhile, six $\beta$-sheet structures at the positions $\beta 1$ : residue $131-134$, position $\beta 2$ : residue $137-143$, position $\beta 3$ : residue $262-263$, position $\beta 4$ : residue $347-$ 352, position $\beta 5$ : residue $355-359$, position $\beta 6$ : residue
487-488. Based on analysis from Some hACE-2 amino acids interact with spike proteins from SARS-CoV-2. The amino acids are Gly447, Tyr449, Gly496, Phe497, Asn501, Arg403, Tyr505, Gly502, Gln506, Thr500, Asn439, Gln498, Glu484, Pro491, Phe486, Ser477, Gly476, Asn487, Tyr489, Lys417, Leu455, and Gln493 (Jin et al. 2020).

\section{Solubility and Lipinski analysis on li- gands}

All ligands that have been optimized for the structure were then analyzed for solubility and permeability. The ligand structure's psychochemical properties can describe the solubility and permeability analysis of a drug candidate. It has been reported that there are five Lipinski's rules that can predict the solubility and permeability of a ligand, which are: 1) a ligand should not have five hydrogen bond donors, 2) a ligand should not have more than 10 hydrogen bond acceptors, 3) a ligand should not have a molecular weight more than $500 \mathrm{Da}, 4$ ) a ligand should not have $\log$ P value less than five, 5) a ligand should not have polar surface area (PSA) more than $140 \mathrm{~A}$ with ligand rotational bonds less than 10 30. This study used four parameters: molecular weight, donor $\mathrm{H}$, acceptor $\mathrm{H}$, and $\log \mathrm{P}$.

With the psychochemical analysis of the ligands used as SARS-CoV-2 inhibitor candidates, it was found that the molecular weights of the ligands analyzed ranged between 100 and 1,200 Da. The smallest molecular weight was $136.23 \mathrm{Da}$, and the largest 1526.45. Also, the log P value ranged between -16 and 18 . The hydrogen bond donor value was between 0 and 29, with the hydrogen bond acceptor value between 0 and 47. Based on the ligand analysis of Lipinski's rule, it was found that 17 ligands were not included in Lipinski's rule, i.e., Methyl 3, 5-di-O-caffeoyl quinate, Proanthocyanidins, Rutin, Corilagin, cyanidin 3-O-sambubioside), delphinidin-3-O-sambubioside, ptilidepsin, remdesivir, Antimycin A, Cadiolide B, Chitosan, Dieckol, EPS (Exopolysaccharide), Mollamide F, Phlorofucofuroeckol A, Prunolide A, Thalassiolin D, and Thalassodendrone.

The ADME data and Lipinski's rule demonstrated that among the top five metabolite compounds obtained based on the Gibbs free energy $(\Delta \mathrm{G})$, namely corilagin, dieckol, phlorofucofuroeckol A, proanthocyanidins A, isovitexin, only isovitexin meets Lipinski's rules. Lipinski’s rule also does not permit the control of Remdesivir and Ptilidepsin, the two compounds that have been used in the treatment of COVID-19. This is interesting as the absence of corilagin, dieckol, phlorofucofuroeckol A, and proanthocyanidins $\mathrm{A}$ is related to molecular sizes larger than $500 \mathrm{Da}$. It was known that corilagin, dieckol, phlorofucofuroeckol A, proanthocyanidins, remdesivir, and ptilidepsin have molecular weights $634.45 \mathrm{Da}, 742.55 \mathrm{Da}, 602.46 \mathrm{Da}, 592.22 \mathrm{Da}$, 1110.34, and 602.58 Da, respectively. Based on Lipinski's analysis, molecular weight $<500 \mathrm{Da}$ is more accessible to mass produce, more stable, easy to apply as an oral drug. Based on this analysis, metabolite compounds can be applied in medicine, such as COVID-19 with further observations on drug delivery and other pharmacological conditions. 
Table 3. Toxicity analysis from secondary metabolites.

\begin{tabular}{|c|c|c|c|c|c|c|c|c|c|c|}
\hline \multirow{2}{*}{$\begin{array}{l}\text { No. } \\
1\end{array}$} & \multirow{2}{*}{$\begin{array}{c}\text { Compound } \\
\text { Neoandrographolide }\end{array}$} & \multicolumn{2}{|c|}{ Acute Oral Toxicity (c) } & \multirow{2}{*}{$\begin{array}{c}\begin{array}{c}\text { Acute Oral } \\
\text { Toxicity }(\mathrm{kg} / \mathrm{mol})\end{array} \\
3.128\end{array}$} & \multicolumn{2}{|c|}{$\begin{array}{l}\text { Carcinogenicity } \\
\text { (binary) }\end{array}$} & \multicolumn{2}{|c|}{ Carcinogenicity (trinary) } & \multicolumn{2}{|c|}{ Hepatotoxicity } \\
\hline & & I & 0.511 & & - & 1 & Non-required & 0.6395 & - & 0.8 \\
\hline 2 & Stigmasterol & & 0.4287 & 3.285 & - & 0.8571 & Non-required & 0.5888 & - & 0.775 \\
\hline 3 & Arisugacin A & & 0.3643 & 3.617 & - & 0.9 & Non-required & 0.5934 & + & 0.575 \\
\hline 4 & Manoalide & & 0.6488 & 2.964 & - & 0.7571 & Non-required & 0.5752 & - & 0.65 \\
\hline 5 & Brazilein & & 0.3586 & 3.068 & - & 0.8775 & Non-required & 0.4864 & + & 0.575 \\
\hline 6 & Atherosperminine & II & 0.481 & 2.936 & - & 0.8571 & Non-required & 0.6356 & + & 0.7 \\
\hline 7 & Coumarin & & 0.7019 & 2.514 & - & 0.9714 & Warning & 0.5324 & - & 0.525 \\
\hline 8 & Quercetin & & 0.7348 & 2.559 & - & 1 & Non-required & 0.675 & + & 0.75 \\
\hline 9 & Alterporriol Q & & 0.575 & 2.419 & - & 0.793 & Non-required & 0.5429 & + & 0.8 \\
\hline 10 & Caffeine & & 0.7405 & 2.138 & - & 0.9429 & Non-required & 0.6936 & + & 0.65 \\
\hline 11 & Chloroquine & & 0.737 & 2.684 & - & 0.8286 & Non-required & 0.6847 & + & 0.55 \\
\hline 12 & Luteolin & & 0.7348 & 2.525 & - & 1 & Non-required & 0.675 & + & 0.825 \\
\hline 13 & Murrangatin & & 0.4572 & 2.293 & - & 0.9714 & Non-required & 0.608 & + & 0.7 \\
\hline 14 & Naringenin & & 0.3682 & 1.87 & - & 0.9857 & Non-required & 0.6152 & + & 0.675 \\
\hline 15 & Eurycomanone & III & 0.5156 & 3.703 & - & 1 & Non-required & 0.6076 & - & 0.55 \\
\hline 16 & Lupeol & & 0.8578 & 3.852 & - & 0.9714 & Non-required & 0.5755 & - & 0.575 \\
\hline 17 & Methyl 3,5-di-O-caffeoyl quinate & & 0.661 & 2.231 & - & 0.8602 & Non-required & 0.585 & + & 0.575 \\
\hline 18 & Proanthocyanidins & & 0.6109 & 2.405 & - & 0.9286 & Non-required & 0.5213 & + & 0.625 \\
\hline 19 & (-) Alphapinene & & 0.8258 & 1.527 & - & 0.7286 & Non-required & 0.4741 & - & 0.75 \\
\hline 20 & (+) Alphaninene & & 0.8258 & 1.527 & - & 0.7286 & Non-required & 0.4741 & - & 0.75 \\
\hline 21 & (E)_cinnamaldehyde & & 0.8687 & 1.485 & - & 0.5075 & Non-required & 0.6995 & - & 0.6 \\
\hline 22 & 2-hydroxycinnamaldehyde & & 0.7966 & 2.446 & - & 0.5816 & Non-required & 0.6488 & + & 0.525 \\
\hline 23 & 6-gingerol & & 0.6007 & 2.29 & - & 0.7 & Non-required & 0.7188 & - & 0.8 \\
\hline 24 & 6-shogaol & & 0.6916 & 2.267 & - & 0.7731 & Non-required & 0.6917 & - & 0.675 \\
\hline 25 & 7-hydroxycoumarin/Umbelliferone & & 0.5546 & 1.759 & - & 1 & Non-required & 0.4671 & - & 0.55 \\
\hline 26 & 7-methoxycoumarin & & 0.8038 & 1.707 & - & 0.9646 & Warning & 0.4614 & - & 0.5 \\
\hline 27 & 14_deoxy_11_12_didehydroandrographolide & & 0.4627 & 2.353 & - & 0.9571 & Non-required & 0.5955 & - & 0.75 \\
\hline 28 & Alpha-curcumene & & 0.9346 & 2.06 & - & 0.7 & Warning & 0.4736 & - & 0.8 \\
\hline 29 & andrographolide & & 0.5328 & 2.795 & - & 0.9714 & Non-required & 0.5856 & - & 0.725 \\
\hline 30 & Anomuricine & & 0.5396 & 2.749 & - & 1 & Non-required & 0.7496 & + & 0.525 \\
\hline 31 & Anomurine & & 0.4952 & 2.032 & - & 1 & Non-required & 0.7406 & + & 0.575 \\
\hline 32 & Artemisidiyne A & & 0.5665 & 1.897 & - & 0.8446 & Non-required & 0.7287 & - & 0.775 \\
\hline 33 & Aurantiamide & & 0.671 & 1.916 & - & 0.8857 & Non-required & 0.7808 & + & 0.55 \\
\hline 34 & Aurantiamide acetate & & 0.6698 & 1.878 & - & 0.8143 & Non-required & 0.7243 & + & 0.625 \\
\hline 35 & Balanophonin & & 0.5707 & 1.659 & - & 0.8571 & Non-required & 0.5429 & + & 0.7 \\
\hline 36 & Beta-sesquiphellandrene & & 0.9084 & 2.444 & - & 0.6714 & Warning & 0.5271 & - & 0.825 \\
\hline 37 & Beta-turmerone & & 0.8277 & 2.851 & - & 0.8286 & Non-required & 0.5778 & - & 0.7 \\
\hline 38 & Caffeic acid ethyl ester & & 0.791 & 2.115 & - & 0.7 & Non-required & 0.663 & - & 0.6 \\
\hline 39 & Camphene & & 0.836 & 2.088 & - & 0.7286 & Non-required & 0.4777 & - & 0.75 \\
\hline 40 & Canthine & & 0.5991 & 2.208 & - & 0.8143 & Non-required & 0.5444 & + & 0.575 \\
\hline 41 & Cinnamic acid & & 0.8487 & 1.672 & - & 0.7571 & Non-required & 0.7458 & - & 0.65 \\
\hline 42 & Cinnamyl alcohol & & 0.902 & 1.327 & + & 0.5714 & Warning & 0.5046 & - & 0.8 \\
\hline 43 & Coclaurine & & 0.5575 & 1.779 & - & 1 & Non-required & 0.7311 & - & 0.575 \\
\hline 44 & Coreximine & & 0.4795 & 1.83 & - & 1 & Non-required & 0.6748 & + & 0.525 \\
\hline 45 & Curcumin & & 0.6349 & 1.992 & - & 0.8061 & Non-required & 0.713 & + & 0.725 \\
\hline 46 & Deoxyandrographolide & & 0.4913 & 2.457 & - & 0.9714 & Non-required & 0.5553 & - & 0.725 \\
\hline 47 & Reticuline & & 0.7348 & 1.477 & - & 1 & Non-required & 0.7169 & - & 0.55 \\
\hline 48 & Rutin & & 0.5971 & 2.593 & - & 0.9857 & Non-required & 0.6741 & + & 0.7 \\
\hline 49 & Stepharine & & 0.49 & 2.491 & - & 0.816 & Non-required & 0.6415 & + & 0.6 \\
\hline 50 & Alpha-turmerone & & 0.6957 & 2.551 & - & 0.8286 & Non-required & 0.5457 & - & 0.7 \\
\hline 51 & Xanthone & & 0.5082 & 1.678 & - & 0.9031 & Warning & 0.482 & + & 0.75 \\
\hline 52 & Corilagin & & 0.4887 & 2.385 & - & 0.9429 & Non-required & 0.7032 & + & 0.7 \\
\hline 53 & Cyanidin 3-O-sambubioside & & 0.4867 & 2.658 & - & 0.9714 & Non-required & 0.6278 & + & 0.575 \\
\hline 54 & Delphinidin-3-O-sambubioside & & 0.4867 & 2.802 & - & 0.9714 & Non-required & 0.6278 & + & 0.575 \\
\hline 55 & Phyllanthin & & 0.6433 & 1.894 & - & 0.8 & Non-required & 0.5028 & - & 0.625 \\
\hline 56 & Blumeatin & & 0.6169 & 1.783 & - & 0.9857 & Non-required & 0.5741 & + & 0.625 \\
\hline 57 & Cordycepin & & 0.7761 & 2.423 & - & 0.9857 & Non-required & 0.5214 & + & 0.825 \\
\hline 58 & Plitidepsin & & 0.6636 & 3.878 & - & 0.8286 & Non-required & 0.6177 & + & 0.6 \\
\hline 59 & Remdesivir & & 0.5357 & 3.428 & - & 0.9714 & Non-required & 0.5361 & + & 0.675 \\
\hline 60 & 3,4-dihydroxybenzoic acid & & 0.5059 & 1.111 & - & 0.6626 & Non-required & 0.6219 & - & 0.625 \\
\hline 61 & Antimycin Ala & & 0.7509 & 2.198 & - & 0.9857 & Non-required & 0.7008 & + & 0.65 \\
\hline 62 & Thalassodendrone & & 0.703 & 1.486 & - & 0.8745 & Non-required & 0.727 & + & 0.675 \\
\hline 63 & Asebotin & & 0.8125 & 2.18 & - & 0.9429 & Non-required & 0.7539 & + & 0.575 \\
\hline 64 & Asperterrestide A & & 0.6853 & 2.687 & - & 0.7857 & Non-required & 0.6545 & + & 0.85 \\
\hline 65 & Bengamide A & & 0.6773 & 2.698 & - & 0.8714 & Non-required & 0.672 & + & 0.525 \\
\hline 66 & Butenolides & & 0.7466 & 1.473 & - & 0.7446 & Non-required & 0.4511 & - & 0.6 \\
\hline 67 & Cadiolide B & & 0.5169 & 3.239 & - & 0.7905 & Danger & 0.6868 & + & 0.725 \\
\hline 68 & Chitosan & & 0.5497 & 2.741 & - & 0.9714 & Non-required & 0.6119 & - & 0.55 \\
\hline 69 & Comaparvin & & 0.6855 & 3.376 & - & 0.9286 & Non-required & 0.6719 & + & 0.75 \\
\hline
\end{tabular}




\begin{tabular}{|c|c|c|c|c|c|c|c|c|c|c|}
\hline \multirow{2}{*}{$\begin{array}{l}\text { No. } \\
70\end{array}$} & \multirow{2}{*}{$\begin{array}{c}\text { Compound } \\
\text { Debromoaplysiatoxin }\end{array}$} & \multicolumn{2}{|c|}{ Acute Oral Toxicity (c) } & \multirow{2}{*}{$\begin{array}{c}\begin{array}{c}\text { Acute Oral } \\
\text { Toxicity }(\mathbf{k g} / \mathbf{m o l})\end{array} \\
3.944\end{array}$} & \multicolumn{2}{|c|}{$\begin{array}{l}\text { Carcinogenicity } \\
\quad \text { (binary) }\end{array}$} & \multicolumn{2}{|c|}{ Carcinogenicity (trinary) } & \multicolumn{2}{|c|}{ Hepatotoxicity } \\
\hline & & III & 0.3458 & & - & 0.9571 & Non-required & 0.6529 & - & 0.5 \\
\hline 71 & Dieckol & & 0.5444 & 2.826 & - & 0.8429 & Non-required & 0.6122 & + & 0.6 \\
\hline 72 & Durumolide J & & 0.6049 & 3.514 & - & 0.9857 & Non-required & 0.5214 & - & 0.65 \\
\hline 73 & Ehrenbergol C & & 0.6183 & 1.915 & - & 0.9143 & Non-required & 0.6495 & - & 0.75 \\
\hline 74 & EPS (Exopolysaccharide) & & 0.5523 & 3.194 & - & 0.9714 & Non-required & 0.6941 & + & 0.55 \\
\hline 75 & Eudistomin C & & 0.5714 & 2.147 & - & 0.9714 & Non-required & 0.5207 & - & 0.575 \\
\hline 76 & Fucoidan & & 0.5961 & 3.171 & - & 0.8286 & Non-required & 0.6466 & - & 0.8 \\
\hline 77 & Furan-2-yl acetate & & 0.8517 & 2.117 & - & 0.7 & Warning & 0.4295 & - & 0.65 \\
\hline 78 & Gyrosanol A & & 0.8909 & 2.781 & - & 0.9714 & Non-required & 0.6699 & - & 0.9 \\
\hline 79 & Metachromin A & & 0.6322 & 2.646 & - & 0.8 & Non-required & 0.6787 & + & 0.525 \\
\hline 80 & MGDG & & 0.592 & 2.282 & - & 0.9857 & Non-required & 0.748 & - & 0.65 \\
\hline 81 & Mollamide F & & 0.6486 & 3.037 & - & 0.7286 & Non-required & 0.6604 & + & 0.7 \\
\hline 82 & Molleurea A & & 0.6651 & 1.643 & - & 0.9 & Non-required & 0.7405 & + & 0.675 \\
\hline 83 & Nortopsentins & & 0.6963 & 1.57 & - & 0.9143 & Non-required & 0.6325 & + & 0.8 \\
\hline 84 & Pateamine A & & 0.621 & 3.292 & - & 0.9 & Non-required & 0.5053 & + & 0.8 \\
\hline 85 & Phlorofucofuroeckol A & & 0.4563 & 2.833 & - & 0.9143 & Non-required & 0.4241 & + & 0.625 \\
\hline 86 & Secocembranoid & & 0.8206 & 1.382 & - & 0.8286 & Non-required & 0.6219 & - & 0.675 \\
\hline 87 & Prunolide A & & 0.5379 & 2.955 & - & 0.8303 & Danger & 0.6776 & + & 0.8 \\
\hline 88 & Stachybogrisephenone B & & 0.51 & 2.047 & - & 0.6622 & Non-required & 0.6082 & + & 0.9 \\
\hline 89 & Thalassiolin D & & 0.5737 & 2.588 & - & 0.9571 & Non-required & 0.6311 & + & 0.7 \\
\hline 90 & Thalassodendrone & & 0.8181 & 1.93 & - & 0.9429 & Non-required & 0.7441 & + & 0.7 \\
\hline 91 & Sulfated polysaccharide & & 0.6868 & 2.032 & - & 0.9571 & Non-required & 0.508 & + & 0.9 \\
\hline 92 & Zoanthoxanthine & & 0.4947 & 1.721 & - & 0.8857 & Danger & 0.566 & + & 0.725 \\
\hline 93 & 2-Acetyl-1-Pyrroline & & 0.6266 & 1.783 & - & 0.7459 & Non-required & 0.6289 & - & 0.725 \\
\hline 94 & Favipiravir & & 0.6291 & 1.78 & - & 0.9286 & Non-required & 0.7394 & + & 0.8 \\
\hline 95 & Gnetin C & & 0.4075 & 2.383 & - & 0.9143 & Danger & 0.3539 & + & 0.725 \\
\hline 96 & Gnetol & & 0.7754 & 2.158 & - & 0.5301 & Non-required & 0.6573 & + & 0.925 \\
\hline 97 & Hematoxyline & & 0.4369 & 3.357 & - & 0.8857 & Warning & 0.5244 & - & 0.575 \\
\hline 98 & Piperine & & 0.8002 & 2.201 & - & 0.9198 & Non-required & 0.5912 & - & 0.5 \\
\hline 99 & Protocatechuic acid & & 0.5059 & 1.111 & - & 0.6626 & Non-required & 0.6219 & - & 0.625 \\
\hline 100 & Terpinen-4-ol & & 0.8213 & 2.047 & - & 0.8429 & Non-required & 0.597 & - & 0.85 \\
\hline 101 & Xanthorrhizol & & 0.8442 & 2.462 & - & 0.6571 & Non-required & 0.6691 & - & 0.725 \\
\hline 102 & Isovitexin & IV & 0.3746 & 2.812 & - & 0.9857 & Non-required & 0.7252 & + & 0.6 \\
\hline 103 & Catechin & & 0.6433 & 2.141 & - & 0.9286 & Non-required & 0.5825 & - & 0.5 \\
\hline 104 & EPA & & 0.6387 & 2.698 & - & 0.6714 & Non-required & 0.6373 & - & 0.65 \\
\hline 105 & Omega-3 & & 0.6387 & 1.22 & - & 0.6714 & Non-required & 0.6373 & - & 0.65 \\
\hline
\end{tabular}

\section{Toxicity analysis with admetSAR}

Ligand toxicity analysis aims to determine the level of toxicity of a ligand using the admetSAR site. This method was used based on the activity-structure relationship used to predict the pharmacokinetic level and toxicity of a ligand. In this study, carcinogenicity (binary and trinary), hepatotoxicity, and acute oral toxicity based on class and ligand concentration were used. The carcinogenicity assessment was based on data on the Carcinogenic Potency Database (CPDB), where there are 1,547 chemical structures with tumor data on rodents that have carcinogenic potential as seen from the TD50 value. The screening method for carcinogenic compounds uses the Morgan fingerprint and the $k$-nearest neighbors $(k \mathrm{NN})$ method. Meanwhile, the hepatotoxicity assessment was based on the DrugBank database with 3,115 toxic compounds and 593 non-toxic compounds. The ligands were prepared using Pipeline Pilot, with the inorganic compounds, large molecular compounds (> $800 \mathrm{Da})$, and inorganic salts in the mixture removed. The acute oral toxicity assessment was based on a database wherein there were 10,207 compounds with LD50 in a mouse model ${ }^{24}$.

Carcinogenicity analysis of compounds using admetSAR was classified into two models, binary and trinary.
The development of these models from admetSAR to predict the carcinogenicity of a compound was accomplished using five machine learning methods, namely support vector machine (SVM), $k \mathrm{NN}$, random forest (RF), C4.5 decision tree (DT), and naïve Bayes (NB), combined with six types of fingerprints. However, the best binary and trinary models were constructed using the $k \mathrm{NN}$ and SVM algorithms with the MACCS fingerprint. The binary classification aims to distinguish chemical compounds with various structures into carcinogenic active and inactive (Lipinski et al. 2004). In contrast, the trinary classification, the three-class classification, aims to predict a chemical compound's carcinogenic potency as non-required, warning, and danger based on the median toxic dose (TD50), the dose required to cause a toxic effect in $50 \%$ of the population (Cheng et al. 2012). If a compound was categorized as non-required, it indicates that the compound is non-carcinogenic; a warning means that the compound is carcinogenic with a TD50 > $10 \mathrm{mg} / \mathrm{kg}$ body weight/ day, while danger implies a carcinogenic compound with a TD50 $\geq 10 \mathrm{mg} / \mathrm{kg}$ body weight/day. In general, the results of the carcinogenicity analysis of compounds using admetSAR with binary model revealed that the majority of compounds are non-carcinogenic, only one out of 105 compounds indicated to be carcinogenic, there is cinna- 
Table 4. Gibbs free energy analysis from molecular docking simulations.

\begin{tabular}{|c|c|c|c|c|c|c|c|}
\hline \multicolumn{8}{|c|}{ Gibbs free energy $(\Delta G)$} \\
\hline Compound & hACE-2 & $\mathrm{M}^{\text {pro }}$ & PL $^{\text {pro }}$ & Compound & hACE-2 & $\mathrm{M}^{\mathrm{pro}}$ & $P^{\text {pro }}$ \\
\hline Corilagin & -10.25 & -9.98 & -8.74 & Gnetol & -6.49 & -5.55 & -5.78 \\
\hline Dieckol & -10.23 & -9.77 & -9.12 & Artemisidiyne A & -6.40 & -5.07 & -5.26 \\
\hline Phlorofucofuroeckol A & -9.73 & -9.43 & -8.43 & Atherosperminine & -6.37 & -5.62 & -5.22 \\
\hline Proanthocyanidins & -9.22 & -7.81 & -8.34 & Gyrosanol A & -6.35 & -5.64 & -6.16 \\
\hline Isovitexin & -9.19 & -7.92 & -8.23 & Xanthone & -6.18 & -5.76 & -5.69 \\
\hline Neoandrographolide & -9.18 & -7.84 & -8.24 & $\begin{array}{l}\text { MGDG (monogalactosyl- } \\
\text { diacylglyceride) }\end{array}$ & -6.17 & -5.12 & -4.83 \\
\hline Lupeol & -9.18 & -7.17 & -8.32 & Anomuricine & -6.13 & -5.14 & -5.45 \\
\hline Prunolide A & -9.16 & -7.73 & -6.73 & Bengamide A & -6.04 & -4.98 & -5.41 \\
\hline Methyl 3, 5-di-O-caffeoyl quinate & -9.15 & -7.88 & -8.26 & Anomurine & -5.85 & -4.83 & -4.83 \\
\hline Eurycomanone & -9.01 & -7.89 & -8.26 & Cordycepin & -5.82 & -5.12 & -5.38 \\
\hline Alterporriol Q & -8.96 & -8.25 & -7.61 & Zoanthoxanthine & -5.81 & -5.15 & -5.68 \\
\hline Cyanidin 3-O-sambubioside & -8.88 & -7.92 & -7.52 & Curcumin & -5.76 & -4.53 & -4.89 \\
\hline Delphinidin-3-O-sambubioside & -8.76 & -7.91 & -7.66 & Phyllanthin & -5.73 & -5.04 & -4.95 \\
\hline Thalassodendrone & -8.65 & -7.91 & -7.25 & Chloroquine & -5.73 & -4.42 & -4.68 \\
\hline Cadiolide B & -8.63 & -7.08 & -7.39 & Alpha-turmerone & -5.59 & -3.90 & -4.86 \\
\hline Chitosan & -8.60 & -6.85 & -6.99 & Secocembranoid & -5.58 & -3.78 & -4.39 \\
\hline Aurantiamide & -8.43 & -6.70 & -6.80 & (+) Alphapinene & -5.55 & -4.61 & -4.84 \\
\hline Aurantiamide acetate & -8.35 & -7.36 & -6.69 & Beta-turmerone & -5.54 & -4.13 & -4.99 \\
\hline Mollamide F & -8.30 & -6.62 & -6.72 & Reticuline & -5.51 & -4.32 & -4.89 \\
\hline Gnetin C & -8.26 & -6.96 & -6.73 & Rutin & -5.51 & -4.20 & -5.00 \\
\hline Thalassiolin D & -8.21 & -7.20 & -6.92 & Alpha-curcumene & -5.50 & -4.39 & -5.45 \\
\hline Molleurea A & -8.21 & -5.87 & -6.37 & Quercetin & -5.50 & -4.21 & -4.79 \\
\hline Andrographolide & -8.17 & -6.85 & -6.84 & Beta-sesquiphellandrene & -5.45 & -4.01 & -4.74 \\
\hline $\begin{array}{l}\text { 14_deoxy_11_12_ } \\
\text { didehydroandrographolide }\end{array}$ & -8.16 & -6.89 & -6.84 & $\mathrm{EPA}$ & -5.37 & -3.57 & -4.04 \\
\hline Arisugacin A & -8.12 & -8.08 & -7.21 & 7-hydroxycoumarin & -5.34 & -4.44 & -4.86 \\
\hline Deoxyandrographolide & -8.05 & -6.66 & -6.35 & 6-shogaol & -5.34 & -3.93 & -4.55 \\
\hline Debromoaplysiatoxin & -7.99 & -6.72 & -6.56 & 7-methoxycoumarin & -5.28 & -4.46 & -4.88 \\
\hline Plitidepsin & -7.93 & -6.12 & -5.14 & Zalcitabine & -5.24 & -4.65 & -5.09 \\
\hline Nortopsentin D (Nortopsentins) & -7.91 & -6.75 & -6.89 & Stepharine & -5.24 & -4.03 & -4.71 \\
\hline Stigmasterol & -7.82 & -6.50 & -6.23 & Coumarin & -5.19 & -4.29 & -4.58 \\
\hline Pateamine A & -7.78 & -6.16 & -5.98 & Xanthorrhizol & -5.19 & -4.02 & -4.76 \\
\hline Asebotin & -7.70 & -6.96 & -6.51 & Omega-3 & -5.18 & -3.33 & -4.12 \\
\hline Remdesivir & -7.68 & -6.73 & -6.05 & 6-gingerol & -5.14 & -3.82 & -4.45 \\
\hline EPS (Exopolysaccharide) & -7.64 & -7.27 & -6.90 & Fucoidan & -4.90 & -4.58 & -4.86 \\
\hline Asperterrestide A & -7.54 & -6.42 & -6.14 & Cinnamic acid & -4.87 & -3.92 & -4.71 \\
\hline Manoalide & -7.52 & -6.19 & -6.39 & Terpinen-4-ol & -4.85 & -4.34 & -4.84 \\
\hline Blumeatin & -7.28 & -6.46 & -6.41 & Catechin & -4.84 & -3.97 & -4.63 \\
\hline Metachromin A & -7.18 & -5.64 & -6.27 & 3,4-dihydroxybenzoic acid & -4.83 & -4.59 & -4.61 \\
\hline Antimycin Ala & -7.09 & -6.10 & -5.97 & Canthine & -4.83 & -3.92 & -4.59 \\
\hline Comaparvin & -7.05 & -5.98 & -6.00 & Protocatechuic acid & -4.79 & -4.20 & -8.34 \\
\hline Brazilein & -6.94 & -6.19 & -6.02 & Caffeic acid ethyl ester & -4.79 & -3.96 & -4.62 \\
\hline Murrangatin & -6.91 & -6.29 & -6.07 & Balanophonin & -4.77 & -3.95 & -4.62 \\
\hline Luteolin & -6.90 & -6.28 & -6.17 & Caffeine & -4.71 & -4.20 & -4.39 \\
\hline Stachybogrisephenone B & -6.84 & -6.04 & -6.02 & 2-hydroxycinnamaldehyde & -4.53 & -4.03 & -4.42 \\
\hline Coreximine & -6.81 & -5.83 & -5.69 & Butenolides & -4.48 & -4.18 & -4.85 \\
\hline $\begin{array}{l}\text { Asebogenin (6'-O-rhamnosyl- } \\
\left(1^{\prime \prime} \rightarrow 6 "\right) \text {-glucopyranosyl }\end{array}$ & -6.73 & -6.08 & -6.52 & Camphene & -4.46 & -3.94 & -4.13 \\
\hline Naringenin & -6.72 & -6.08 & -5.97 & (-)Alphapinene & -4.42 & -7.81 & -4.08 \\
\hline Eudistomin C & -6.71 & -5.22 & -6.19 & Cinnamyl alcohol & -4.30 & -3.38 & -3.97 \\
\hline Durumolide J & -6.70 & -6.20 & -5.87 & (E)_cinnamaldehyde & -4.28 & -3.42 & -3.86 \\
\hline Ehrenbergol C & -6.68 & -6.15 & -5.85 & Furan-2-yl acetate & -4.07 & -3.72 & -4.62 \\
\hline Piperine & -6.55 & -4.94 & -5.50 & 2-Acetyl-1-Pyrroline & -3.64 & -3.30 & -3.93 \\
\hline Coclaurine & -6.54 & -5.17 & -5.56 & Favipiravir & -0.91 & -0.75 & -0.98 \\
\hline Hematoxyline & -6.50 & -5.51 & -5.81 & & & & \\
\hline
\end{tabular}

myl alcohol (No. 32). In the carcinogenicity analysis results using the binary model, the non-carcinogenic compounds were marked with a negative sign (-) with a range of probability or accuracy values between 0.5075 and 1 . In contrast, the compounds predicted to be carcinogenic have a positive sign (+) with a probability value of 0.5714 .

Besides the carcinogenicity analysis, hepatotoxicity analysis is vital in drug discovery and development efforts because liver toxicity is at the top of drug reduction. The hepatotoxicity analysis was performed using admetSAR to classify the compounds into active/ inactive or positive/negative (Morris et al. 1998). The analysis revealed that 56 out of the 105 compounds were predicted to be hepatotoxic agents with probability values between 0.525 and 0.925 . Meanwhile, the other 49 compounds were predicted to be non-hepatotoxic with their probability values between 0.5 and 0.9 .

The acute oral toxicity analysis for classifying compounds into four categories based on the value of $50 \%$ lethal dose (LD50), generally expressed in terms of the amount of material per unit of body weight ( $\mathrm{Lu}$ et al. 
Table 5. Receptor and ligand interactions based on hydrogen bond analysis.

\begin{tabular}{|c|c|c|c|}
\hline Reseptor & ACE-2 ( $)$ & $\mathbf{M}^{\text {pro }}(\AA)$ & $\mathbf{P L}^{\text {pro }}(\AA)$ \\
\hline Ligan & & & \\
\hline \multirow[t]{7}{*}{ Dieckol } & Tyr $515(3.25)$ & Met $276(3.25)$ & Tyr 274 (3.38), (3.13) \\
\hline & Gln $442(3.08)$ & Leu 287 (3.32) & Thr $302(3.13)$ \\
\hline & Glu 406 (3.34) & Tyr 239 (2.88) & Leu 163 (3.02), (2.92) \\
\hline & $\operatorname{Arg} 518$ (3.33), (3.03) & Asn 238 (2.98) & \\
\hline & Ser $409(2.93)$ & Asp 197 (3.08), (3.16) & \\
\hline & His 437 (3.04) & Thr $169(3.08)$ & \\
\hline & Thr 337 (3.02), (3,04) & Thr 135 (3.16) & \\
\hline \multirow[t]{6}{*}{ Isovitexin } & Gln $442(3.35)$ & Leu 287 (3.28) & Thr 75 (3.06), (2.78) \\
\hline & Lys 44 (3.08) & Leu $272(3.27)$ & Leu 76 (2.96) \\
\hline & Ser $409(2.98)$ & Asp 289 (3.12) & Asp 77 (3.10) \\
\hline & Arg $518(2.81),(2.93),(3.08)$ & Arg $131(3.07)$ & Gln 175 (3.25) \\
\hline & & Thr 199 (3.03) & Asn 157 (3.06) \\
\hline & & Asn 238 (2.97), (3.30) & \\
\hline \multirow[t]{6}{*}{ Proanthocyanidins } & Ser 409 (3.03) & Leu $287(3.28)$ & Thr 75(3.06), (2.79) \\
\hline & Lys 441 (3.08) & Leu 272 (3.28) & Leu $76(2.92)$ \\
\hline & Arg $518(2.84),(2.94),(3.10)$ & Asp 289 (3.11) & Asp 77 (3.09) \\
\hline & & Arg 131 (3.09) & Gln $175(3.22)$ \\
\hline & & Thr $199(3.03)$ & Asn 157 (3.01) \\
\hline & & Asn 238 (3.30), (2.98) & \\
\hline \multirow[t]{5}{*}{ Corilagin } & Ser $43(2.98)$ & Lys $137(2.96)$ & Thr 75 (3.06), (2.78) \\
\hline & Asp 350 (3.15) & Arg $131(3.31)$ & Leu $76(2.96)$ \\
\hline & Arg $393(3.00)$ & Asp $197(3.36)$ & Asp 77 (3.10) \\
\hline & Tyr 385 (3.13), (3.10) & Leu 287 (3.07), (2.84) & Gln $175(3.25)$ \\
\hline & & & Asn 157 (3.06) \\
\hline \multirow[t]{7}{*}{ Phlorofucofuroeckol A } & Ser $43(2.80)$ & Thr $169(2.70)$ & Leu $76(2.92)$ \\
\hline & Asp 350 (3.28) & Thr 135 (2.99) & Thr 75 (2.83), (3.06) \\
\hline & Ala 348 (3.09) & Asp 197 (3.23) & Tyr $155(3.17)$ \\
\hline & Asp 67 (3.30) & Leu 287 (3.67). (3.14) & Asn 157 (3.11) \\
\hline & Ser 70 (2.99) & Asn 238 (3.19) & His 176 (3.02) \\
\hline & & Thr 199 (3.22), (2.89). (3.14) & \\
\hline & & Tyr $237(3.24)$ & \\
\hline \multirow[t]{4}{*}{ Ptilidepsin } & Ser 105 (3.15), (3.06) & Met $276(2.96)$ & Met $209(3.11)$ \\
\hline & Tyr $202(2.70)$ & & $\operatorname{Gln} 175(3.01)$ \\
\hline & Gln 102 (3.15), (3.23) & & \\
\hline & $\operatorname{Trp} 69(3.16)$ & & \\
\hline \multirow[t]{3}{*}{ Remdesivir } & Asp 350 (3.15) & Thr 199 (3.05), (3.02), (2.93) & - \\
\hline & Asn 394 (2.96) & & \\
\hline & Arg 514 (3.15) & & \\
\hline
\end{tabular}

2009), a dose or concentration of a substance/compound estimated to have caused the death of half of the individuals who receive it. The categorization of toxicity refers to the United States Environmental Protection Agency (U.S. EPA) that classifies chemicals into four categories with different toxicity levels. The four categories of acute oral toxicity are category I (danger/poison), II (warning), III (caution), and IV (non-required). The compounds falling under category I are those with LD50 $\leq 50 \mathrm{mg} / \mathrm{kg}$, under category II are compounds with LD50 $>50 \mathrm{mg} / \mathrm{kg}$ and $\leq$ $500 \mathrm{mg} / \mathrm{kg}$, under category III are compounds with LD50 $>500 \mathrm{mg} / \mathrm{kg}$ and $\leq 5,000 \mathrm{mg} / \mathrm{kg}$, and under category IV are the compound with LD50 value $>5,000 \mathrm{mg} / \mathrm{kg}$. In other words, compounds with category I have the highest acute oral toxicity among other categories (Lipinski et al. 2004; Ho et al. 2005). The selected 105 compounds belonged to various acute oral toxicity categories: category I (five compounds), category II (nine compounds), category III (87 compounds), and category IV (four compounds).

The docking results show that there are five best compounds for potential drugs from the total 105 compounds: isovitexin, proanthocyanidins, corilagin, and dieckol phlorofucofuroeckol A. The toxicity analysis results reveal that these five compounds are non-carcinogenic, even though they can be hepatotoxic agents with a value of $<$ $75 \%$. These five compounds' acute oral toxicity also indicated that they were classified as category I (isovitexin) and category III (proanthocyanidins, corilagin, dieckol, phlorofucofuroeckol A compounds).

\section{Gibbs free energy analysis}

Based on the Gibbs free energy analysis, the more negative the value resulting from molecular docking simulation, the more stable the bond between ligands and receptors. Therefore, the ligands with a stable negative energy value against the three receptors $\mathrm{ACE}-2, \mathrm{M}^{\mathrm{pro}}$, and $\mathrm{PL}^{\text {pro }}$ will be discussed. The ligands to be discussed are those with the best five Gibbs free energy values $(\Delta \mathrm{G})$ : corilagin, dieckol, phlorofucofuroeckol A, proanthocyanidins, isovitexin, with ptilidepsin and remdesivir as controls (common drugs) used as comparators (Table 3 and Suppl. material 1: Figure S1). Based on the analysis of interactions between ligands and receptors, it can be deduced that several bonds were formed between the two, such as hydrophobic bonds, van der Waals bonds, and hydrogen bonds.

The analysis of hydrogen bonds revealed that ACE-2 receptors have interactions with metabolite compounds 
with several amino acid residues, such as Gln 442, Arg 518, Ser 409, Lys 441, Arg 518, and Asp 350. As for amino acid residues Leu 287, Asn 238, Thr 199, and Leu 272, Asn 238 mostly interacted with metabolite compounds on $\mathrm{M}^{\text {pro }}$ receptors. Meanwhile, amino acid residues $\mathrm{Thr}$ 75, Leu 76, Asp 77, Gln 175, and Asn 157 are the most residues that interact with metabolite compounds (Table 4). The longest hydrogen bond was detected for Leu
287 at the $\mathrm{M}^{\text {pro }}$ receptor, with a length of $3.67 \AA$, and the shortest for Thr 169 at the same receptor, with a length $2.70 \AA$ A Meanwhile, the conformation, interaction, and orientation of dieckol and ptylidepsin were illustrated in Figure 4. Dieckol is in the receptor-binding site region, allowing dieckol to have the potential as an inhibitor between the receptor and SARS-CoV-2 spike protein, similar for the ptylidepsin control.

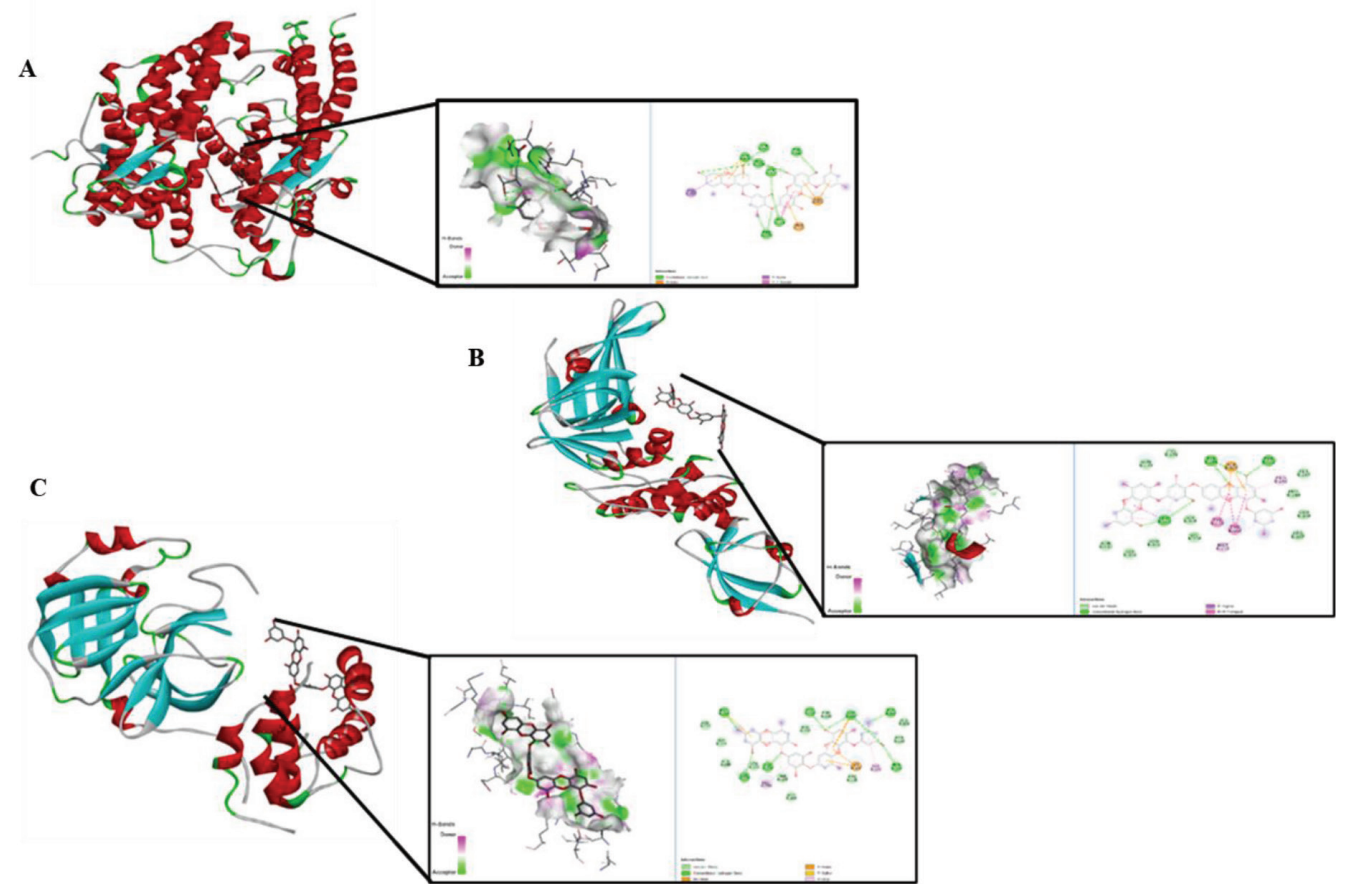

Figure 4. Analysis of the conformation and interaction of hydrogen bonds between ligands and receptors with a distance $<5 \AA$. A. ACE-2-dieckol; B. PL ${ }^{\text {pro }}$-dieckol; C. $\mathrm{M}^{\text {pro-dieckol. }}$

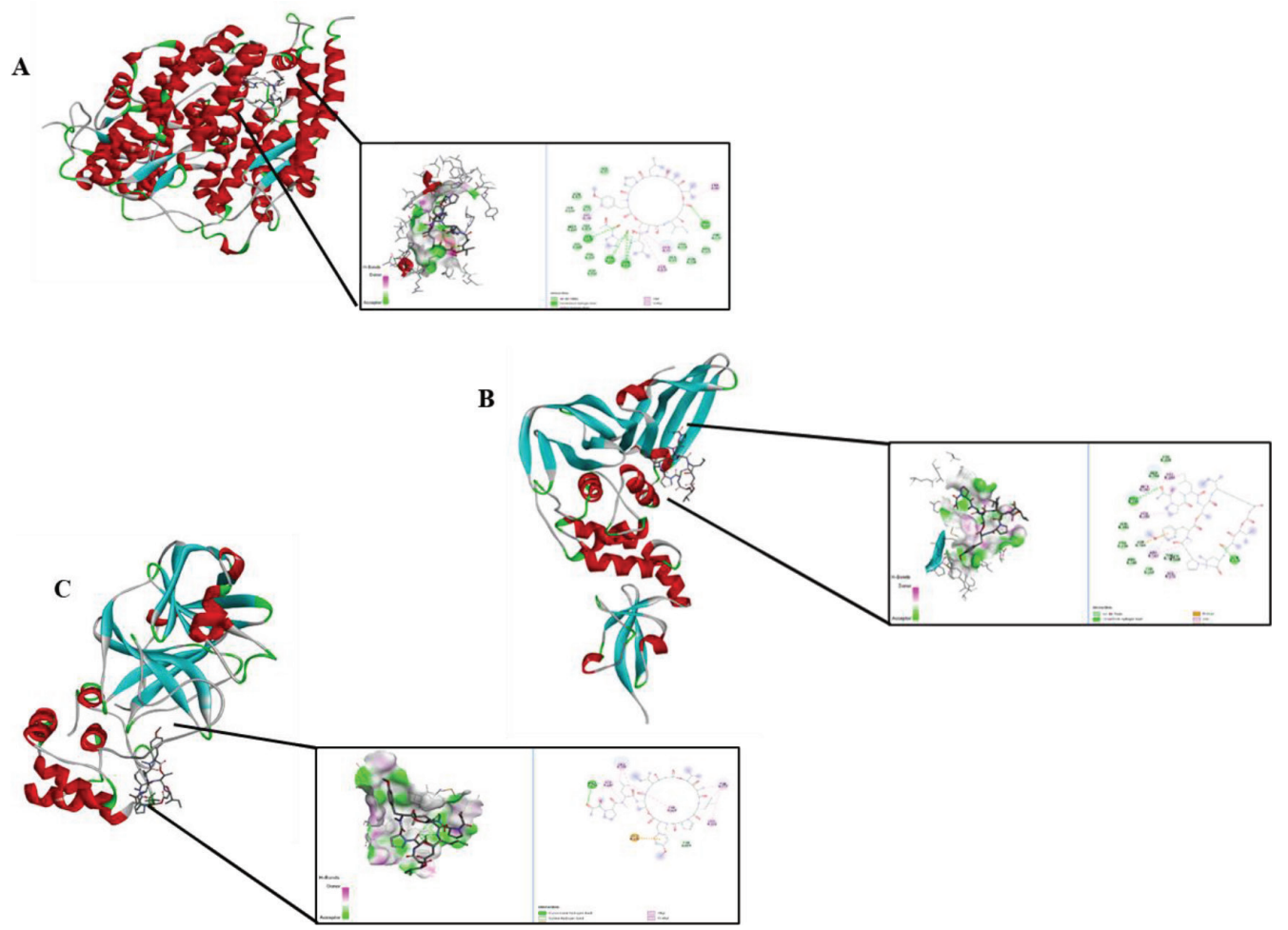

Figure 5. Analysis of the conformation and interaction of hydrogen bonds between ligands and receptors with a distance $<5 \AA$. A. ACE-2-ptilidepsin; B. PL ${ }^{\text {pro }}$-ptilidepsin; C. $\mathrm{M}^{\text {pro }}$-ptilidepsin. 


\section{Conclusion}

Through molecular docking, toxicity, ADME, and Lipinski, virtual screening has been successfully performed on 102 secondary metabolites compounds against three important receptors in SARS CoV-2; ACE-2, $\mathrm{M}^{\text {pro }}$, PL ${ }^{\text {pro }}$. The analysis obtained that corilagin, dieckol, phlorofucofuroeckol A, proanthocyanidins, and isovitexin can inhibit all three receptors. The study of hydrogen bonds revealed that ACE-2 receptors have interactions with metabolite compounds with several amino acid residues, such as Gln 442, Arg 518, Ser 409, Lys 441, Arg 518, and Asp 350. As for amino acid residues Leu 287, Asn 238, Thr 199, and Leu 272, Asn 238 mostly interacted with metabolite compounds on $\mathrm{M}^{\text {pro }}$ receptors. Meanwhile, amino acid residues Thr 75, Leu 76, Asp 77, Gln 175, and Asn 157 are the most residues that interact with metabolite

\section{References}

Abdul Wahab SM, Jantan I, Haque MA, Arshad L (2018) Exploring the leaves of Annona muricata L. as a source of potential anti-inflammatory and anticancer agents. Frontiers in Pharmacology 9: e661. https://doi.org/10.3389/fphar.2018.00661

Abu-Ghefreh AA, Canatan H, Ezeamuzie CI (2009) In vitro and in vivo anti-inflammatory effects of andrographolide. International Immunopharmacology 9(3): 313-318. https://doi.org/10.1016/j.intimp.2008.12.002

Akinwumi BC, Bordun K-AM, Anderson HD (2018) Biological activities of stilbenoids. International Journal of Molecular Sciences 19(3). https://doi.org/10.3390/ijms19030792

Beigel JH, Tomashek KM, Dodd LE, Mehta AK, Zingman BS, Kalil AC, Hohmann E, Chu HY, Luetkemeyer A, Kline S, de Castilla DL, Finberg RW, Dierberg K, Tapson V, Hsieh L, Patterson TF, Paredes R Sweeney DA, Short WR, Touloumi G, Lye DC, Ohmagari N, Oh M-d, Ruiz-Palacios GM, Benfield T, Fätkenheuer G, Kortepeter MG, Atmar RL, Creech CB, Lundgren J, Babiker AG, Pett S, Neaton JD Burgess TH, Bonnett T, Green M, Makowski M, Osinusi A, Nayak S, Lane HC, for the ACTT-1 Study Group Members (2020) Remdesivir for the treatment of Covid-19 - final report. The New England Journal of Medicine 383(19): 1813-1826. https://doi.org/10.1056/ NEJMoa2007764

Benítez-Cardoza CG, Vique-Sánchez JL (2020) Potential Inhibitors of the Interaction between ACE2 and SARS-CoV-2 (RBD) to Develop a Drug. Life Sciences 256: e117970. https://doi.org/10.1016/j. lfs.2020.117970

Bento EB, Matias EF, Brito Jr FE, Oliveira DR, Coutinho HD, Costa JGM, Kerntopf MR, Menezes IRA (2013) Association between food and drugs: antimicrobial and synergistic activity of Annona muricata L. International Journal of Food Properties 16(4): 738-744. https://doi. org/10.1080/10942912.2011.565905

Boulangé A, Parraga J, Galán A, Cabedo N, Leleu S, Sanz MJ, Franck X (2015) Synthesis and antibacterial activities of cadiolides A, B and $\mathrm{C}$ and analogues. Bioorganic \& Medicinal Chemistry 23(13): 36183628. https://doi.org/10.1016/j.bmc.2015.04.010

Brackman G, Defoirdt T, Miyamoto C, Bossier P, Van Calenbergh S, Nelis H, Coenye T (2008) Cinnamaldehyde and cinnamaldehyde derivatives reduce virulence in Vibrio spp. by decreasing the DNA-bind- in $\mathrm{PL}^{\text {pro. }}$. These five compounds have conformation and orientation in the binding site receptors. We recommend the five compounds be developed as drug candidates in the treatment to decrease the growth of the SARS CoV-2 virus. Molecular docking results can be used as a scientific basis in selecting drug candidates before being done in the laboratory.

\section{Acknowledgements}

This research was funded by the Indonesia Endowment Fund for Education (LPDP), with contract number 86/FI/ PKS-KCOVID-19.E/VI/2020. Authors greatly acknowledge to the Head of Research Center for Biotechnology, Indonesian Institute of Sciences, as well as the research and administration staff members for their support.

ing activity of the quorum sensing response regulator LuxR. BMC microbiology 8(1): 1-14. https://doi.org/10.1186/1471-2180-8-149

Charles DJ (2012) Sources of natural antioxidants and their activities. Antioxidant Properties of Spices, Herbs and Other Sources: 65-138. https://doi.org/10.1007/978-1-4614-4310-0_4

Chen B-J, Fu C-S, Li G-H, Wang X-N, Lou H-X, Ren D-M, Shen T (2017) Cinnamaldehyde analogues as potential therapeutic agents. Mini Reviews in Medicinal Chemistry 17(1): 33-43. https://doi.org /10.2174/1389557516666160121120744

Chen L-C, Lin Y-Y, Jean Y-H, Lu Y, Chen W-F, Yang S-N, Wang H-MD, Jang I-Y, Chen I-M, Su J-H, Sung P-J, Sheu J-H, Wen Z-H (2014) Anti-inflammatory and analgesic effects of the marine-derived compound comaparvin isolated from the crinoid Comanthus bennetti. Molecules (Basel, Switzerland) 19(9): 14667-14686. https://doi. org/10.3390/molecules190914667

Cheng F, Li W, Zhou Y, Shen J, Wu Z, Liu G, Lee PW, Tang Y (2019) Correction to "admetSAR: A Comprehensive Source and Free Tool for Assessment of Chemical ADMET Properties". Journal of Chemical Information and Modelling 59(11): e4959. https://doi.org/10.1021/ acs.jcim.9b00969

Cheng S-Y, Chuang C-T, Wang S-K, Wen Z-H, Chiou S-F, Hsu C-H, Dai C-F, Duh C-Y (2010) Antiviral and anti-inflammatory diterpenoids from the soft coral Sinularia gyrosa. Journal of Natural Products 73(6): 1184-1187. https://doi.org/10.1021/np100185a

Cheng S-Y, Wang S-K, Duh C-Y (2014) Secocrassumol, a seco-cembranoid from the Dongsha Atoll soft coral Lobophytum crassum. Marine Drugs 12(12): 6028-6037. https://doi.org/10.3390/md12126028 Cheng S-Y, Wen Z-H, Wang S-K, Chiou S-F, Hsu C-H, Dai C-F, Duh C-Y (2009) Anti-inflammatory cembranolides from the soft coral Lobophytum durum. Bioorganic \& Medicinal Chemistry 17(11): 3763-3769. https://doi.org/10.1016/j.bmc.2009.04.053

Chensom S, Shimada Y, Nakayama H, Yoshida K, Kondo T, Katsuzaki H, Hasegawa S, Mishima T (2020) Determination of anthocyanins and antioxidants in "titanbicus" edible flowers in vitro and in vivo. Plant Foods for Human Nutrition (Dordrecht, Netherlands) 75(2): 265-271. https://doi.org/10.1007/s11130-020-00813-3

Cheriyan BV, Kadhirvelu P, Nadipelly Jr J, Shanmugasundaram J, Sayeli V, Subramanian V (2017) Anti-nociceptive Effect of 7-methoxy Cou- 
marin from Eupatorium Triplinerve vahl (Asteraceae). Pharmacognosy Magazine 13(49): 81-84.

Cheung RCF, Wong JH, Pan WL, Chan YS, Yin CM, Dan XL, Wang HX, Fang EF, Lam SK, Ngai PHK, Xia LX, Liu F, Ye XY, Zhang GQ, Liu QH, Sha O, Lin P, Ki C, Bekhit AA, Bekhit AE-D, Wan DCC, Ye XJ, Xia J, Ng TB (2014) Antifungal and antiviral products of marine organisms. Applied Microbiology and Biotechnology 98(8): 34753494. https://doi.org/10.1007/s00253-014-5575-0

Chung C-Y, Liu C-H, Wang G-H, Jassey A, Li C-L, Chen L, Chen L, Yen M-H, Lin C-C, Lin L-T (2016) (4R,6S)-2-Dihydromenisdaurilide is a Butenolide that Efficiently Inhibits Hepatitis C Virus Entry. Scientific Reports 6: e29969. https://doi.org/10.1038/srep29969

Daczkowski CM, Dzimianski JV, Clasman JR, Goodwin O, Mesecar AD, Pegan SD (2017) Structural Insights into the Interaction of Coronavirus Papain-like Proteases and Interferon-Stimulated Gene Product 15 from Different Species. Journal of Molecular Biology 429(11): 1661-1683. https://doi.org/10.1016/j.jmb.2017.04.011

Dai X-Q, Cai W-T, Wu X, Chen Y, Han F-M (2017) Protocatechuic acid inhibits hepatitis $\mathrm{B}$ virus replication by activating ERK1/2 pathway and down-regulating HNF4 $\alpha$ and HNF1 $\alpha$ in vitro. Life Sciences 180: 68-74. https://doi.org/10.1016/j.lfs.2017.05.015

Dalan R, Bornstein SR, El-Armouche A, Rodionov RN, Markov A, Wielockx B, Beuschlein F, Boehm BO (2020) The ACE-2 in COVID-19: Foe or Friend?. Hormon and Metabolic Research 52(5): 257-263. https://doi.org/10.1055/a-1155-0501

Das P, Majumder R, Mandal M, Basak P (2020) In-Silico Approach for Identification of Effective and Stable Inhibitors for COVID-19 Main Protease $\left(\mathrm{M}^{\mathrm{pro}}\right)$ from Flavonoid Based Phytochemical Constituents of Calendula Officinalis. Journal of Biomolecular Structure and Dynamics. https://doi.org/10.1080/07391102.2020.1796799

Eom S-H, Moon S-Y, Lee D-S, Kim H-J, Park K, Lee E-W, Kim TH, Chung Y-H, Lee M-S, Kim Y-M (2015) In vitro antiviral activity of dieckol and phlorofucofuroeckol-A isolated from edible brown alga Eisenia bicyclis against murine norovirus. Algae (Korean Phycological Society) 30(3): 241-246. https://doi.org/10.4490/algae.2015.30.3.241

Essien EE, Newby JS, Walker TM, Setzer WN, Ekundayo O (2015) Chemotaxonomic characterization and in-vitro antimicrobial and cytotoxic activities of the leaf essential oil of Curcuma longa grown in southern Nigeria. Medicines (Basel, Switzerland) 2(4): 340-349. https://doi.org/10.3390/medicines2040340

Fakih TM (2020) Dermaseptin-Based Antiviral Peptides to Prevent COVID-19 through in Silico Molecular Docking Studies against SARS-CoV-2 Spike Protein. Pharmaceutical Sciences and Research 7(4): 65-70. https://doi.org/10.7454/psr.v7i4.1079

Liu XF, Guan YL, Yang DZ, Li Z, Yao KD (2001) Antibacterial action of chitosan and carboxymethylated chitosan. Journal of Applied Polymer Science 79(7): 1324-1335. https://doi.org/10.1002/10974628(20010214)79:7<1324::AID-APP210>3.0.CO;2-L

Gabay O, Sanchez C, Salvat C, Chevy F, Breton M, Nourissat G, Wolf C, Jacques C, Berenbaum F (2010) Stigmasterol: a phytosterol with potential anti-osteoarthritic properties. Osteoarthritis and Cartilage 18(1): 106-116. https://doi.org/10.1016/j.joca.2009.08.019

Gao C-H, Wang Y-F, Li S, Qian P-Y, Qi S-H (2011) Alkaloids and sesquiterpenes from the South China Sea gorgonian Echinogorgia pseudossapo. Marine Drugs 9(11): 2479-2487. https://doi.org/10.3390/md9112479

Gautam MK, Gangwar M, Nath G, Rao CV, Goel RK (2012) In-vitro antibacterial activity on human pathogens and total phenolic, flavonoid contents of Murraya paniculata Linn. leaves. Asian Pacific Journal of Tropical Biomedicine 2(3): S1660-S1663. https://doi.org/10.1016/ S2221-1691(12)60472-9

Gill BS, Navgeet, Qiu F (2020) Technologies for extraction and production of bioactive compounds. Biotechnological Production of Bioactive Compounds: 1-36. https://doi.org/10.1016/B978-0-444-64323$0.00001-1$

Goc A, Niedzwiecki A, Rath M (2021). Polyunsaturated $\omega-3$ fatty acids inhibit ACE2-controlled SARS-CoV-2 binding and cellular entry. Scientific Reports 11(1): e5207. https://doi.org/10.1038/s41598-02184850-1

Gogineni V, Schinazi RF, Hamann MT (2015) Role of marine natural products in the genesis of antiviral agents. Chemical Reviews 115(18): 9655-9706. https://doi.org/10.1021/cr4006318

González-Almela E, Sanz MA, García-Moreno M, Northcote P, Pelletier J, Carrasco L (2015) Differential action of pateamine A on translation of genomic and subgenomic mRNAs from Sindbis virus. Virology 484: 41-50. https://doi.org/10.1016/j.virol.2015.05.002

Gupta DK, Kaur P, Leong ST, Tan LT, Prinsep MR, Chu JJH (2014) Anti-Chikungunya viral activities of aplysiatoxin-related compounds from the marine cyanobacterium Trichodesmium erythraeum. Marine Drugs 12(1): 115-127. https://doi.org/10.3390/md12010115

Hamaguchi T, Ono K, Yamada M (2010) REVIEW: Curcumin and Alzheimer's disease: Curcumin and AD. CNS Neuroscience \& Therapeutics, 16(5): 285-297. https://doi.org/10.1111/j.1755-5949.2010.00147.x

Hao T, Yang Y, Li N, Mi Y, Zhang G, Song J, Liang Y, Xiao J, Zhou D, He D, Hou Y (2020) Inflammatory mechanism of cerebral ischemia-reperfusion injury with treatment of stepharine in rats. Phytomedicine: International Journal of Phytotherapy and Phytopharmacology 79: e153353. https://doi.org/10.1016/j.phymed.2020.153353

Hart PH, Brand C, Carson CF, Riley TV, Prager RH, Finlay-Jones JJ (2000) Terpinen-4-ol, the main component of the essential oil of Melaleuca alternifolia (tea tree oil), suppresses inflammatory mediator production by activated human monocytes. Et al [Inflammation Research] 49(11): 619-626. https://doi.org/10.1007/s000110050639

Hawas UW, Abou El-Kassem LT (2017) Thalassiolin D: a new flavone O-glucoside Sulphate from the seagrass Thalassia hemprichii. Natural Product Research 31(20): 2369-2374. https://doi.org/10.1080/14 786419.2017.1308367

Hayashi K, Lee J-B, Atsumi K, Kanazashi M, Shibayama T, Okamoto K, Kawahara T, Hayashi T (2019) In vitro and in vivo anti-herpes simplex virus activity of monogalactosyl diacylglyceride from Coccomyxa sp. KJ (IPOD FERM BP-22254), a green microalga. PLoS ONE 14(7): e0219305. https://doi.org/10.1371/journal.pone.0219305

He F, Bao J, Zhang X-Y, Tu Z-C, Shi Y-M, Qi S-H (2013) Asperterrestide A, a cytotoxic cyclic tetrapeptide from the marine-derived fungus Aspergillus terreus SCSGAF0162. Journal of Natural Products 76(6): 1182-1186. https://doi.org/10.1021/np300897v

Ho BK, Brasseur R (2005) The Ramachandran Plots of Glycine and Pre-Proline. BMC Structural Biology 5(1): 1-14. https://doi. org/10.1186/1472-6807-5-14

Hosseini M, Chen W, Xiao D, Wang C (2021) Computational Molecular Docking and Virtual Screening Revealed Promising SARSCoV-2 Drugs. Precision Clinical Medicine 4(1): 1-16. https://doi. org/10.1093/pcmedi/pbab001

Hung TM, Na MK, Thuong PT, Su ND, Sok DE, Song KS, Seong YH, Baea KH (2006) Antioxidant activity of caffeoyl quinic acid derivatives from the roots of Dipsacus asper Wall. Journal of Ethnopharmacology 108(2): 188-192. https://doi.org/10.1016/j.jep.2006.04.029 
Hwang JK, Shim JS, Baek NI, Pyun YR (2000) Xanthorrhizol: a potential antibacterial agent from Curcuma xanthorrhiza against Streptococcus mutans. Planta Medica 66(2): 196-197. https://doi. org/10.1055/s-0029-1243135

Ibrahim MAA, Abdeljawaad KAA, Abdelrahman AHM, Hegazy MEF (2020) Natural-like Products as Potential SARS-CoV-2 Mpro Inhibitors: In-Silico Drug Discovery. Journal of Biomolecular Structure and Dynamics. https://doi.org/10.1080/07391102.2020.1790037

Ishii H, Koyama H, Hagiwara K, Miura T, Xue G, Hashimoto Y, Hashimoto Y, Kitahara G, Aida Y, Suzuki M (2012) Synthesis and biological evaluation of deoxy-hematoxylin derivatives as a novel class of anti-HIV-1 agents. Bioorganic \& Medicinal Chemistry Letters 22(3) 1469-1474. https://doi.org/10.1016/j.bmcl.2011.06.066

Ji X, Guo J, Liu Y, Lu A, Wang Z, Li Y, Yang S, Wang Q (2018) Marine-natural-product development: First discovery of nortopsentin alkaloids as novel antiviral, anti-phytopathogenic-fungus, and insecticidal agents. Journal of Agricultural and Food Chemistry 66(16) 4062-4072. https://doi.org/10.1021/acs.jafc.8b00507

Jiang Y, Wu N, Fu Y-J, Wang W, Luo M, Zhao C-J, Zu Y-G, Liu X-L (2011) Chemical composition and antimicrobial activity of the essential oil of Rosemary. Environmental Toxicology and Pharmacology 32(1): 63-68. https://doi.org/10.1016/j.etap.2011.03.011

Jin Z, Du X, Xu Y, Deng Y, Liu M, Zhao Y, Zhang B, Li X, Zhang L, Peng C, Duan Y, Yu J, Wang L, Yang K, Liu F, Jiang R, Yang X, You T, Liu X, Yang X, Bai F, Liu H, Liu X, Guddat LW, Xu W, Xiao G, Qin C, Shi Z, Jiang H, Rao Z, Yang H (2020) Structure of $M^{\text {pro }}$ from SARS-CoV-2 and Discovery of Its Inhibitors. Nature 582(7811): 289-293. https:// doi.org/10.1038/s41586-020-2223-y

Joshi T, Sharma P, Joshi T, Pundir H, Mathpal, S, Chandra S (2020) Structure-Based Screening of Novel Lichen Compounds against SARS Coronavirus Main Protease ( $\left.\mathrm{M}^{\text {pro }}\right)$ as Potentials Inhibitors of COVID-19. Molecular Diversity. https://doi.org/10.1007/s11030-020-10118-X

Kamdem RE, Sang S, Ho C-T (2002) Mechanism of the superoxide scavenging activity of neoandrographolide - a natural product from Andrographis paniculata Nees. Journal of Agricultural and Food Chemistry 50(16): 4662-4665. https://doi.org/10.1021/jf025556f

Kim TH, Ku S-K, Bae J-S (2012) Antithrombotic and profibrinolytic activities of eckol and dieckol. Journal of Cellular Biochemistry 113(9): 2877-2883. https://doi.org/10.1002/jcb.24163

Kinoshita S, Inoue Y, Nakama S, Ichiba T, Aniya Y (2007) Antioxidant and hepatoprotective actions of medicinal herb, Terminalia catappa L. from Okinawa Island and its tannin corilagin. Phytomedicine: International Journal of Phytotherapy and Phytopharmacology 14(11): 755-762. https://doi.org/10.1016/j.phymed.2006.12.012

Kochuthressia K, Britto S (2012) In vitro antimicrobial evaluation of Kaempferia galanga L. rhizome extract. American Journal of Biotechnology and Molecular Sciences 2(1): 1-5. https://doi.org/10.5251/ ajbms.2012.2.1.1.5

Krithika R, Jyothilakshmi V, Verma RJ (2016) Phyllanthin inhibits $\mathrm{CCl} 4$-mediated oxidative stress and hepatic fibrosis by down-regulating TNF- $\alpha / N F-\kappa B$, and pro-fibrotic factor TGF- $\beta 1$ mediating inflammatory signaling. Toxicology and Industrial Health 32(5); 953-960. https://doi.org/10.1177/0748233714532996

Kulprachakarn K, Pangjit K, Paradee N, Srichairatanakool S, Rerkasem K, Ounjaijean S (2019) Antioxidant properties and cytotoxicity of white mugwort (Artemisia lactiflora) leaf extract in human hepatocellular carcinoma cell line. Walailak Journal of Science and Technology 16(3): 185-192. https://doi.org/10.48048/wjst.2019.6223
Kumar A, Dholakia K, Sikorska G, Martinez LA, Levenson AS (2019) MTA1-dependent anticancer activity of Gnetin C in prostate cancer. Nutrients 11(9): e2096. https://doi.org/10.3390/nu11092096

Kuno F, Otoguro K, Shiomi K, Iwai Y, Omura S (1996) Arisugacins A and $\mathrm{B}$, novel and selective acetylcholinesterase inhibitors from Penicillium sp. FO-4259. I. Screening, taxonomy, fermentation, isolation and biological activity. The Journal of Antibiotics 49(8): 742-747. https:// doi.org/10.7164/antibiotics.49.742

Kwon H-J, Ryu YB, Kim Y-M, Song N, Kim CY, Rho M-C, Jeong J-H, Cho K-O, Lee WS, Park S-J (2013) In vitro antiviral activity of phlorotannins isolated from Ecklonia cava against porcine epidemic diarrhea coronavirus infection and hemagglutination. Bioorganic \& Medicinal Chemistry 21(15): 4706-4713. https://doi.org/10.1016/j. bmc.2013.04.085

Lan J, Ge J, Yu J, Shan S, Zhou H, Fan S, Zhang Q, Shi X, Wang Q, Zhang L, Wang X (2020) Structure of the SARS-CoV-2 Spike Receptor-Binding Domain Bound to the ACE2 Receptor. Nature 581(7807): 215220. https://doi.org/10.1038/s41586-020-2180-5

Leboeuf M, Legueut C, Cavé A, Desconclois JF, Forgacs P, Jacquemin H (1981) Alcaloïdes des Annonacées XXIX: Alcaloïdes de l'Annona muricata L. Planta medica 42(1): 37-44. https://doi. org/10.1055/s-2007-971543

Li X, Chen L, Cheng F, Wu Z, Bian H, Xu C, Li W, Liu G, Shen X Tang Y (2014) In Silico Prediction of Chemical Acute Oral Toxicity Using Multi-Classification Methods. Journal of Chemical Information and Modelling 54(4): 1061-1069. https://doi.org/10.1021/ci5000467

Li B, Lu F, Wei X, Zhao R (2008) Fucoidan: structure and bioactivity. Molecules (Basel, Switzerland) 13(8): 1671-1695. https://doi. org/10.3390/molecules13081671

Li S (2011) Chemical composition and product quality control of turmeric (Curcuma longa L.). Pharmaceutical Crops 5(1): 28-54. https://doi.org/10.2174/2210290601102010028

Lim CS, Jin D-Q, Mok H, Oh SJ, Lee JU, Hwang JK, Ha I, Han J-S (2005) Antioxidant and antiinflammatory activities of xanthorrhizol in hippocampal neurons and primary cultured microglia. Journal of Neuroscience Research 82(6): 831-838. https://doi.org/10.1002/ jnr.20692

Lipinski CA (2004) Lead- and Drug-Like Compounds: The Rule-of-Five Revolution. Drug Discovery Today: Technol 1(4): 337-341. https:// doi.org/10.1016/j.ddtec.2004.11.007

Liu YC, Kuo RL, Shih SR (2020) COVID-19: The first documented coronavirus pandemic in history. Biomedical Journal 43(4): 328-333. https://doi.org/10.1016/j.bj.2020.04.007

Liu A-L, Shu S-H, Qin H-L, Lee SMY, Wang Y-T, Du G-H (2009) In vitro anti-influenza viral activities of constituents from Caesalpinia sappan. Planta Medica 75(4): 337-339. https://doi. org/10.1055/s-0028-1112208

Liu J, Wang Z-T, Ji L-L (2007) In vivo and in vitro anti-inflammatory activities of neoandrographolide. The American Journal of Chinese Medicine 35(2): 317-328. https://doi.org/10.1142/S0192415X07004849

Lozano I, Wacyk JM, Carrasco J, Cortez-San Martín MA (2016) Red macroalgae Pyropia columbina and Gracilaria chilensis: sustainable feed additive in the Salmo salar diet and the evaluation of potential antiviral activity against infectious salmon anemia virus. Journal of Applied Phycology 28(2): 1343-1351. https://doi.org/10.1007/ s10811-015-0648-8

Lu HM, Yin DC, Ye YJ, Luo HM, Geng LQ, Li HS, Guo WH, Shang P (2009) Correlation between Protein Sequence Similarity and X-Ray 
Diffraction Quality in the Protein Data Bank. Protein and Peptide Letter 16(1): 50-55. https://doi.org/10.2174/092986609787049457

Lu Z, Harper MK, Pond CD, Barrows LR, Ireland CM, Van Wagoner RM (2012) Thiazoline peptides and a tris-phenethyl urea from Didemnum molle with anti-HIV activity. Journal of Natural Products 75(8): 1436-1440. https://doi.org/10.1021/np300270p

Machhi J, Herskovitz J, Senan AM, Dutta D, Nath B, Oleynikov MD, Blomberg WR, Meigs DD, Hasan M, Patel M, Kline P, Chang RCC, Chang L, Gendelman HE, Kevadiya BD (2020) The Natural History, Pathobiology, and Clinical Manifestations of SARS-CoV-2 Infections. Journal of Neuroimmune Pharmacology 15(3): 359-386. https://doi.org/10.1007/s11481-020-09944-5

Malinowska M, Sikora E, Ogonowski J (2015) Lipophilicity of lupeol semisynthetic derivates. Prace Naukowe Uniwersytetu Ekonomicznego We Wrocławiu (411). https://doi.org/10.15611/pn.2015.411.08

Mishra K, Dash AP, Dey N (2011) Andrographolide: A novel antimalarial diterpene lactone compound from Andrographis paniculata and its interaction with curcumin and artesunate. Journal of Tropical Medicine: e579518. https://doi.org/10.1155/2011/579518

Mohammed MMD, Hamdy A-HA, El-Fiky NM, Mettwally WSA, El-Beih AA, Kobayashi N (2014) Anti-influenza A virus activity of a new dihydrochalcone diglycoside isolated from the Egyptian seagrass Thalassodendron ciliatum (Forsk.) den Hartog. Natural Product Research 28(6): 377-382. https://doi.org/10.1080/14786419.2013.869694

Mori Y, Ono T, Miyahira Y, Nguyen VQ, Matsui T, Ishihara M (2013) Antiviral activity of silver nanoparticle/chitosan composites against H1N1 influenza A virus. Nanoscale Research Letters 8(1): e93. https://doi.org/10.1186/1556-276X-8-93

Morita H, Kishi E, Takeya K, Itokawa H, Tanaka O (1990) New Quassinoids from the Roots ofEurycoma longifolia. Chemistry Letters, 19(5): 749-752. https://doi.org/10.1246/cl.1990.749

Morris GM, Goodsell DS, Halliday RS, Huey R, Hart WE, Belew RK, Olson AJ (1998) Automated Docking Using a Lamarckian Genetic Algorithm and an Empirical Binding Free Energy Function. Journal of Computational Chemistry 19(14): 1639-1662. https://doi. org/10.1002/(SICI) 1096-987X(19981115)19:14<1639::AID-JCC10>3.0.CO;2-B

Muhammad DRA, Tuenter E, Patria GD, Foubert K, Pieters L, Dewettinck K (2021) Phytochemical composition and antioxidant activity of Cinnamomum burmannii Blume extracts and their potential application in white chocolate. Food Chemistry 340: e127983. https:// doi.org/10.1016/j.foodchem.2020.127983

Mynderse JS, Moore RE, Kashiwagi M, Norton TR (1977) Antileukemia activity in the Osillatoriaceae: isolation of Debromoaplysiatoxin from Lyngbya. Science (New York, N.Y.) 196(4289): 538-540. https:// doi.org/10.1126/science.403608

Naidoo D, Roy A, Kar P, Mutanda T, Anandraj A (2020) Cyanobacterial Metabolites as Promising Drug Leads against the Mpro and PLpro of SARS-CoV-2: An in Silico Analysis. Journal of Biomolecular Structure and Dynamics. https://doi.org/10.1080/07391102.2020.1794972

Nakagami Y, Suzuki S, Espinoza JL, Vu Quang L, Enomoto M, Takasugi S, Nakamura A, Nakayama T, Tani H, Hanamura I, Takami A (2019) Immunomodulatory and metabolic changes after Gnetin- $C$ supplementation in humans. Nutrients 11(6): e1403. https://doi. org/10.3390/nu11061403

Nakamura Y, Kawamoto N, Ohto Y, Torikai K, Murakami A, Ohigashi H (1999) A diacetylenic spiroketal enol ether epoxide, AL-1, from Artemisia lactiflora inhibits 12-O-tetradecanoylphorbol-13-ace- tate-induced tumor promotion possibly by suppression of oxidative stress. Cancer Letters 140(1-2): 37-45. https://doi.org/10.1016/ S0304-3835(99)00048-8

Nor FM, Mohamed S, Idris NA, Ismail R (2008) Antioxidative properties of Pandanus amaryllifolius leaf extracts in accelerated oxidation and deep frying studies. Food Chemistry 110(2): 319-327. https://doi. org/10.1016/j.foodchem.2008.02.004

Nunnari G, Argyris E, Fang J, Mehlman KE, Pomerantz RJ, Daniel R (2005) Inhibition of HIV-1 replication by caffeine and caffeine-related methylxanthines. Virology 335(2): 177-184. https://doi. org/10.1016/j.virol.2005.02.015

Ooi JP, Kuroyanagi M, Sulaiman SF, Muhammad TST, Tan ML (2011) Andrographolide and 14-deoxy-11, 12-didehydroandrographolide inhibit cytochrome P450s in HepG2 hepatoma cells. Life Sciences, 88(9-10): 447-454. https://doi.org/10.1016/j.lfs.2010.12.019

Oon SF, Nallappan M, Tee TT, Shohaimi S, Kassim NK, Sa’ariwijaya MSF, Cheah YH (2015) Xanthorrhizol: a review of its pharmacological activities and anticancer properties. Cancer Cell International 15(1): e100. https://doi.org/10.1186/s12935-015-0255-4

Orhan IE, Deniz FSS (2020) Natural Products as Potential Leads against Coronaviruses: Could They Be Encouraging Structural Models against SARS-CoV-2?. Natural Products and Bioprospecting 10(4): 171-186. https://doi.org/10.1007/s13659-020-00250-4

Ota Y, Chinen T, Yoshida K, Kudo S, Nagumo Y, Shiwa Y, Yamada R, Umihara H, Iwasaki K, Masumoto H, Yokoshima S, Yoshikawa H, Fukuyama T, Kobayashi J, Usui T (2016) Eudistomin C, an antitumor and antiviral natural product, targets $40 \mathrm{~S}$ ribosome and inhibits protein translation. Chembiochem: A European Journal of Chemical Biology 17(17): 1616-1620. https://doi.org/10.1002/cbic.201600075

Ou CB, Pan Q, Chen X, Hou N, He C (2012) Protocatechuic acid, a new active substance against the challenge of avian infectious bursal disease virus. Poultry Science 91(7): 1604-1609. https://doi. org/10.3382/ps.2011-02069

Plumb GW, De Pascual-Teresa S, Santos-Buelga C, Cheynier V, Williamson G (1998) Antioxidant properties of catechins and proanthocyanidins: effect of polymerisation, galloylation and glycosylation. Free Radical Research 29(4): 351-358. https://doi. org/10.1080/10715769800300391

Qin C, Lin X, Lu X, Wan J, Zhou X, Liao S, Tu Z, Xu S, Liu Y (2015) Sesquiterpenoids and xanthones derivatives produced by sponge-derived fungus Stachybotry sp. HH1 ZSDS1F1-2. The Journal of Antibiotics 68(2): 121-125. https://doi.org/10.1038/ja.2014.97

Raveh A, Delekta PC, Dobry CJ, Peng W, Schultz PJ, Blakely PK, Tai AW, Matainaho T, Irani DN, Sherman DH, Miller DJ (2013) Discovery of potent broad spectrum antivirals derived from marine Actinobacteria. PLoS ONE 8(12): e82318. https://doi.org/10.1371/journal. pone. 0082318

Remsberg CM, Martinez SE, Akinwumi BC, Anderson HD, Takemoto JK, Sayre CL, Davies NM (2015) Preclinical pharmacokinetics and pharmacodynamics and content analysis of gnetol in foodstuffs: Pharmacokinetics and pharmacodynamics of gnetol. Phytotherapy Research: PTR 29(8): 1168-1179. https://doi.org/10.1002/ptr.5363

Rohman A, Widodo H, Lukitaningsih E, Rafi M, Nurrulhidayah, Windarsih A (2019) Review on in vitro antioxidant activities of Curcuma species commonly used as herbal components in Indonesia. Food Research 4(2): 286-293. https://doi.org/10.26656/fr.2017.4(2).163

Ryu YB, Jeong HJ, Yoon SY, Park J-Y, Kim YM, Park S-J, Rho M-C, Kim S-J, Lee WS (2011) Influenza virus neuraminidase inhibitory activity 
of phlorotannins from the edible brown alga Ecklonia cava. Journal of Agricultural and Food Chemistry 59(12): 6467-6473. https://doi org/10.1021/jf2007248

Salam KA, Furuta A, Noda N, Tsuneda S, Sekiguchi Y, Yamashita A, Moriishi K, Nakakoshi M, Tsubuki M, Tani H, Tanaka J, Akimitsu N (2013) Psammaplin A inhibits hepatitis C virus NS3 helicase. Journal of Natural Medicines 67(4): 765-772. https://doi.org/10.1007/ s11418-013-0742-7

Shereen MA, Khan S, Kazmi A, Bashir N, Siddique R (2020) COVID-19 Infection: Emergence, Transmission, and Characteristics of Human Coronaviruses. Journal of Advanced Research 24: e91. https://doi. org/10.1016/j.jare.2020.03.005

Sitanggang BR, Prijanti AR, Astuty H (2018) The role of pasak bumi (Eurycoma longifolia Jack) extract as an antimalarial agent through the mechanism of antioxidant specific activity (superoxide dismutase, SOD and catalase, CAT) in Plasmodium berghei-infected mice. Advanced Science Letters 24(9): 6976-6979. https://doi.org/10.1166/ asl.2018.12900

Smitha D, Kumar MMK, Ramana H, Rao DV (2014) Rubrolide R: a new furanone metabolite from the ascidian Synoicum of the Indian Ocean. Natural Product Research 28(1): 12-17. https://doi.org/10.10 80/14786419.2013.827194

Sogo T, Kumamoto T, Ishida H, Hisanaga A, Sakao K, Terahara N, Wada K, Hou D-X (2015) Comparison of the inhibitory effects of delphinidin and its glycosides on cell transformation. Planta Medica 81(1): 26-31. https://doi.org/10.1055/s-0034-1383311

Sogo T, Terahara N, Hisanaga A, Kumamoto T, Yamashiro T, Wu S, Sakao K, Hou D-X (2015) Anti-inflammatory activity and molecular mechanism of delphinidin 3-sambubioside, a Hibiscus anthocyanin: Anti-Inflammatory Effects of Delphinidin 3-Sambubioside. BioFactors (Oxford, England) 41(1): 58-65. https://doi.org/10.1002/biof.1201

Soni VK, Yadav DK, Bano N, Dixit P, Pathak M, Maurya R, Sahai M, Jain SK, Misra-Bhattacharya S (2012) N-Methyl-6, 7-dimethoxyisoquinolone in Annona squamosa twigs is the major immune modifier to elicit polarized Th1 immune response in BALB/c mice. Fitoterapia 83(1): 110-116. https://doi.org/10.1016/j.fitote.2011.09.019

Sova M (2012) Antioxidant and antimicrobial activities of cinnamic acid derivatives. Mini reviews in medicinal chemistry 12(8): 749-767. https://doi.org/10.2174/138955712801264792

Sunila ES, Kuttan G (2004) Immunomodulatory and antitumor activity of Piper longum Linn. and piperine. Journal of Ethnopharmacology 90(2-3): 339-346. https://doi.org/10.1016/j.jep.2003.10.016

Tadych M, White JF (2019) Endophytic Microbes. Reference Module in Life Sciences. https://doi.org/10.1016/B978-0-12-809633-8.13036-5

Tan L, Song X, Ren Y, Wang M, Guo C, Guo D, Gu Y, Li Y, Cao Z, Deng Y (2020) Anti-inflammatory effects of cordycepin: A review. Phytotherapy Research: PTR 35(3): 1284-1297. https://doi.org/10.1002/ ptr.6890

Tang X, Wu C, Li X, Song Y, Yao X, Wu X, Duan Y, Zhang H, Wang Y, Qian Z, Cui J, Lu J (2020) On the origin and continuing evolution of SARS-CoV-2. National. Scientific Review 7: 1012-1023. https://doi. org/10.1093/nsr/nwaa036

Terstappen GC, Reggiani A (2001) In Silico Research in Drug Discovery. Trends in Pharmacological Sciences 22(1): 23-26. https://doi. org/10.1016/S0165-6147(00)01584-4

Tietjen I, Williams DE, Read S, Kuang XT, Mwimanzi P, Wilhelm E, Markle T, Kinloch NN, Naphen CN, Tenney K, Mesplède T, Wainberg MA, Crews P, Bell B, Andersen RJ, Brumme ZL, Brockman MA
(2018) Inhibition of NF- $\mathrm{B}$-dependent HIV-1 replication by the marine natural product bengamide A. Antiviral Research 152: 94-103. https://doi.org/10.1016/j.antiviral.2018.02.017

Tosepu R, Gunawan J, Effendy DS, Ahmad LOAI, Lestari H, Bahar H, Asfian P (2020) Correlation between Weather and Covid-19 Pandemic in Jakarta, Indonesia. Science of the Total Environment 725: e138436. https://doi.org/10.1016/j.scitotenv.2020.138436

Towler P, Staker B, Prasad SG, Menon S, Tang J, Parsons T, Ryan D, Fisher M, Williams D, Dales NA, Patane MA, Pantoliano MW (2004) ACE2 X-Ray Structures Reveal a Large Hinge-Bending Motion Important for Inhibitor Binding and Catalysis. Journal of Biological Chemistry 279(17): 17996-18007. https://doi.org/10.1074/jbc.M311191200

Trivedi NP, Rawal UM, Patel BP (2007) Hepatoprotective effect of andrographolide against hexachlorocyclohexane-induced oxidative injury. Integrative Cancer Therapies 6(3): 271-280. https://doi. org/10.1177/1534735407305985

Trott O, Olson AJ (2010) AutoDock Vina: Improving the Speed and Accuracy of Docking with a New Scoring Function, Efficient Optimization, and Multithreading. Journal of Computational Chemistry 31(2): 455-461. https://doi.org/10.1002/jcc.21334

Tutunchi H, Naeini F, Ostadrahimi A, Hosseinzadeh-Attar MJ (2020) Naringenin, a flavanone with antiviral and anti-inflammatory effects: A promising treatment strategy against COVID-19. Phytotherapy Research: PTR 34(12): 3137-3147. https://doi.org/10.1002/ptr.6781

Tyagi AK, Prasad S, Yuan W, Li S, Aggarwal BB (2015) Identification of a novel compound ( $\beta$-sesquiphellandrene) from turmeric (Curcuma longa) with anticancer potential: comparison with curcumin. Investigational New Drugs 33(6): 1175-1186. https://doi.org/10.1007/ s10637-015-0296-5

Venugopala KN, Rashmi V, Odhav B (2013) Review on natural coumarin lead compounds for their pharmacological activity. BioMed Research International 2013: e963248. https://doi.org/10.1155/2013/963248

Wang L, Sang S, Su M, Wang S, Li H (2020) Tea flavonoids blocking multiple SARS-CoV-2 protein targets judged from molecular docking. https://doi.org/10.21203/rs.3.rs-122589/v1

Wang L-X, Zhao W-H, Lu Y-F, Wang C-X (2019) Antioxidant and cytotoxic activities of distillates purified by means of molecular distillation from ginger extract obtained with supercritical CO2 fluid. Chemistry \& Biodiversity 16(11): e1900357. https://doi.org/10.1002/ cbdv.201900357

Wang S-K, Hsieh M-K, Duh C-Y (2013) New diterpenoids from soft coral Sarcophyton ehrenbergi. Marine Drugs 11(11): 4318-4327. https:// doi.org/10.3390/md11114318

Wang W, Kim H, Nam S-J, Rho BJ, Kang H (2012) Antibacterial butenolides from the Korean tunicate Pseudodistoma antinboja. Journal of Natural Products 75(12): 2049-2054. https://doi.org/10.1021/ np300544a

Wang Z, Hu X, Li Y, Mou X, Wang C, Chen X, Tan Y, Wu C, Liu H, $\mathrm{Xu} H$ (2019). Discovery and SAR research for antivirus activity of novel butenolide on influenza A virus $\mathrm{H} 1 \mathrm{~N} 1$ in vitro and in vivo. ACS Omega 4(8): 13265-13269. https://doi.org/10.1021/acsomega.9b01421

White KM, Rosales R, Yildiz S, Kehrer T, Miorin L, Moreno E, Jangra S, Uccellini MB, Rathnasinghe R, Coughlan L, Martinez-Romero C, Batra J, Rojc A, Bouhaddou M Fabius JM, Obernier K, Dejosez M, Guillén MJ, Losada A, Avilés P, Schotsaert M, Zwaka T, Vignuzzi M, Shokat KM, Krogan NJ, García-Sastre A (2021) Plitidepsin has potent preclinical efficacy against SARS-CoV-2 by targeting the 
host protein eEF1A. Science (New York, N.Y.) 371(6532): 926-931. https://doi.org/10.1126/science.abf4058

Yamashita A, Tamaki M, Kasai H, Tanaka T, Otoguro T, Ryo A, Maekawa S, Enomoto N, de Voogd NJ, Tanaka J, Moriishi K (2017) Inhibitory effects of metachromin A on hepatitis B virus production via impairment of the viral promoter activity. Antiviral Research 145: 136-145. https://doi.org/10.1016/j.antiviral.2017.08.001

Yang X, Zhang H, Zhang Y, Zhao H, Dong A, Xu D, Yang L, Ma Y, Wang $J$ (2010) Analysis of the essential oils of pine cones of Pinus koraiensis Steb. Et Zucc. And P. sylvestris L. From China. Journal of Essential Oil Research 22(5): 446-448. https://doi.org/10.1080/10412905.201 0.9700368

Yap BK, Lee CY, Choi SB, Kamarulzaman EE, Hariono M, Wahab HA (2019) In Silico Identification of Novel Inhibitors. In: Encyclopedia of Bioinformatics and Computational Biology. Elsevier, 761-779. https://doi.org/10.1016/B978-0-12-809633-8.20158-1

Yeo S-G, Song JH, Hong E-H, Lee B-R, Kwon YS, Chang S-Y, Kim SH, Lee SW, Park J-H, Ko H-J (2015) Antiviral effects of Phyllanthus urinaria containing corilagin against human enterovirus 71 and Coxsackievirus A16 in vitro. Archives of Pharmacal Research 38(2): 193-202. https://doi.org/10.1007/s12272-014-0390-9

Yildiz H, Karatas N (2018) Microbial exopolysaccharides: Resources and bioactive properties. Process Biochemistry (Barking, London, England) 72: 41-46. https://doi.org/10.1016/j.procbio.2018.06.009

Yoon SY, Park SJ, Park YJ (2018) The anticancer properties of cordycepin and their underlying mechanisms. International Journal of Molecular Sciences 19(10): e3027. https://doi.org/10.3390/ijms19103027

Yoopan N, Thisoda P, Rangkadilok N, Sahasitiwat S, Pholphana N, Ruchirawat S, Satayavivad J (2007) Cardiovascular effects of 14-deoxy-11,12-didehydroandrographolide and Andrographis paniculata extracts. Planta Medica 73(6): 503-511. https://doi. org/10.1055/s-2007-967181

Zhao J, Zhang J-S, Yang B, Lv G-P, Li S-P (2010) Free radical scavenging activity and characterization of sesquiterpenoids in four species of Curcuma using a TLC bioautography assay and GC-MS analysis. Molecules (Basel, Switzerland) 15(11): 7547-7557. https://doi. org/10.3390/molecules15117547

Zhao Y, Yang G, Ren D, Zhang X, Yin Q, Sun X (2011) Luteolin suppresses growth and migration of human lung cancer cells. Molecular Biology Reports 38(2): 1115-1119. https://doi.org/10.1007/s11033010-0208-x

Zheng C-J, Shao C-L, Guo Z-Y, Chen J-F, Deng D-S, Yang K-L, Chen Y-Y, Fu X-M, She Z-G, Lin Y-C, Wang C-Y (2012) Bioactive hydro- anthraquinones and anthraquinone dimers from a soft coral-derived Alternaria sp. fungus. Journal of Natural Products 75(2): 189-197. https://doi.org/10.1021/np200766d

Zhao J, Zhang J-S, Yang B, Lv G-P, Li S-P (2010) Free radical scavenging activity and characterization of sesquiterpenoids in four species of Curcuma using a TLC bioautography assay and GC-MS analysis. Molecules (Basel, Switzerland) 15(11): 7547-7557. https://doi. org/10.3390/molecules15117547

Zhou X, Meyer CU, Schmidtke P, Zepp F (2002) Effect of cordycepin on interleukin-10 production of human peripheral blood mononuclear cells. European Journal of Pharmacology 453(2-3): 309-317. https:// doi.org/10.1016/S0014-2999(02)02359-2

Zmudzinski M, Rut W, Olech K, Granda J, Giurg M, Burda-Grabowska M, Zhang L, Sun X, Lv Z, Nayak D, Kesik-Brodacka M, Olsen SK, Hilgenfeld R, Drag M (2020) Ebselen Derivatives Are Very Potent Dual Inhibitors of SARS-CoV-2 Proteases - PL pro and $\mathrm{M}^{\text {pro }}$ in in Vitro Studies. bioRxiv. https://doi.org/10.1101/2020.08.30.273979

\section{Supplementary material 1}

\section{Figure $\mathbf{S 1}$}

Authors: Gita Syahputra, Nunik Gustini, Bustanussalam Bustanussalam, Yatri Hapsari, Martha Sari, Ardi Ardiansyah, Asep Bayu, Masteria Yunovilsa Putra

Data type: Image

Explanation note: The analysis of the conformation and interaction of hydrogen bonds between ligands and receptors with a distance $<5 \AA$ A. A. ACE-2-remdesivir, B. PL pro - remdesivir; C. $\mathrm{M}^{\text {pro }}$ - remdesivir, D. ACE-2-isovitexin, E. PL ${ }^{\text {pro }}$ - isovitexin, F. $\mathrm{M}^{\text {pro }}$ - isovitexin, G. ACE 2-corilagin, H. PL pro - corilagin, I. $\mathrm{M}^{\text {pro }}$ - corilagin, J. ACE-2- Phlorofucofuroeckol A, K. PL pro Phlorofucofuroeckol A, L. M ${ }^{\text {pro }}$ - Phlorofucofuroeckol A, M. ACE-2- Proanthocyanidins, N. PL pro - Proanthocyanidins, O. $\mathrm{M}^{\text {pro }}$ - Proanthocyanidins.

Copyright notice: This dataset is made available under the Open Database License (http://opendatacommons.org/licenses/ odbl/1.0). The Open Database License (ODbL) is a license agreement intended to allow users to freely share, modify, and use this Dataset while maintaining this same freedom for others, provided that the original source and author(s) are credited.

Link: https://doi.org/10.3897/pharmacia.68.e68432.suppl1 